\title{
Theoretical Considerations in the Estimation of the Signal-to-Noise Crossover Dose
}

\author{
by
}

Shintaro Hagiwara

A thesis submitted to the Faculty of Graduate and Postdoctoral Affairs

in partial fulfillment of the requirements for the degree of

Master of Science

in

Probability and Statistics

Carleton University

Ottawa, Ontario

(C) 2014

Shintaro Hagiwara 


\section{Acknowledgements}

It would not have been possible to complete this thesis without the help and support of the people around me. Especially I would like to express my deepest gratitude to my supervisor Prof. Patrick Farrell, for his excellent guidance with thoughtful advices, warm encouragement, and editing assistance. A very special thanks goes to my co-supervisor Prof. Daniel Krewski who introduced me to the topic, and provided insightful comments from both statistical and epidemiological perspectives.

I would like to acknowledge the technical support of Drs. Salomon Sand and Chris Portier, for their permission to use the dataset, which enabled me to understand the topic in much greater detail.

I would like to thank the members of my thesis examination committee Profs. Mohamedou Ould Haye, Jason Nielsen, Paul Villeneuve, and Tim Ramsay, for their comments and suggestions.

I owe my greatest appreciation to my family for the support and love they provided me through my entire life. Also, I would like to thank my host parents Kim and Bernard Berry for their support and care ever since the first day I entered Canada. 


\begin{abstract}
A goal of health risk assessment is the determination of the reference dose: an estimate of the exposure level for the human population, which is free from appreciable risk of deleterious effects. The estimation of the point of departure: a dose level at which the incease in risk is thought to be acceptable/tolerable, plays an important role in the derivation of the reference dose. The method of benchmark dose has been widely employed in the determination of the point of departure. However, due to some practical constraints, the method sometimes faces difficulty in determining an appropriate increase in risk that meets the definition of the reference dose. As an alternative, the method of the signal-to-noise crossover dose (SNCD) was introduced in 2011. One of the main objectives here is to develop an optimal design for the SNCR: an additional risk associated with the SNCD. The feasibility of the optimal design is also investigated under different dose-response relationships.
\end{abstract}




\section{Contents}

$\begin{array}{ll}\text { Acknowledgements } & \text { ii }\end{array}$

Abstract

List of Figures $\quad$ vi

List of Tables vii

1 Introduction 1

1.1 Derivation of the endpoints for setting human exposure guide-

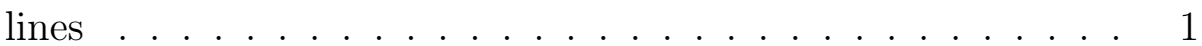

1.2 Methods for deriving a point of departure . . . . . . . . . . 2

1.2.1 The method of NOAEL . . . . . . . . . 2

1.2.2 The method of BMD . . . . . . . . . . . . 3

1.2.3 The method of SNCD . . . . . . . . . . . 4

1.3 The SNCD and its implications . . . . . . . . . . . 6

1.3.1 The SNR . . . . . . . . . . . . . . . . 6

1.3.2 Twisting the concept of SNCD . . . . . . . . . . 7

1.4 The objectives of this study . . . . . . . . . . . . . 9

1.5 The Hill model . . . . . . . . . . . . . . . . . . . . . . . . . . 9

1.6 Four types of dose-response relationships . . . . . . . . . . . 11

2 Developing Optimal Designs for SNCR 12

2.1 MLEs and Optimal Design for BMD/BMR . . . . . . . . . 12

2.1.1 Derivation of the MLE and covariance matrix for the Hill model parameters . . . . . . . . . . . . . . . . 12 
2.1.2 MLEs of BMR and BMD . . . . . . . . . . . . 14

2.1.3 Optimal design for BMD and BMR . . . . . . . . 15

2.2 Search of an explicit solution for SNCD from a given experiment 18

2.3 Optimal designs for SNCD and SNCR . . . . . . . . . . . 19

2.4 Optimal Design for SNCR . . . . . . . . . . . . . . 21

3 Feasibility Study for Optimal Designs 22

3.1 Parameters used in the study . . . . . . . . . . . . . . . . 22

3.2 Process of obtaining optimal designs for SNCR . . . . . . . . . 23

3.3 Results . . . . . . . . . . . . . . . . . . . 24

3.4 Relationship between $\frac{d_{2}}{d_{3}}$ and $\eta$ under optimal designs . . . . . 28

3.5 Sub-optimal designs and their performance . . . . . . . . 30

3.5.1 How to evaluate the performance of a sub-optimal design for SNCR? . . . . . . . . . . . . . . . 30

3.6 Four sub-optimal designs considered in this study . . . . . . . 32

3.6.1 Results and their implications on the performances of sub-optimal designs . . . . . . . . . . . . . 33

3.6.2 The effect of the sample size on $\mathrm{SNCR}_{\text {opt }} \ldots \ldots . . .37$

4 Optimal designs and the NTP database 40

4.1 An Illustrative Example from the NTP database . . . . . . . . 40

5 Conclusions and Discussions

References 
A.1 The Newton-Raphson method for estimating the Hill model parameters . . . . . . . . . . . . . . . . . . . . 49

A.2 Number of dose levels required for optimal designs for BMR and $\mathrm{SNCR} \ldots \ldots \ldots \ldots \ldots \ldots$

A.3 The Identifiability and the Regularity Conditions . . . . . . . 57 A.3.1 Identifiability . . . . . . . . . . . . . . 57

A.3.2 Regularity conditions . . . . . . . . . . . . 58

A.4 Profile Likelihood based Confidence Interval for $\hat{P}(d) \ldots$. . . 63

$\begin{array}{ll}\text { B Definitions } & 65\end{array}$

C List of Acronyms $\quad 66$ 


\section{List of Figures}

1 Four types of dose-response relationship under the Hill model 10

2 Optimal SNCR versus $\alpha(\mathrm{N}=180) \ldots \ldots . \ldots 26$

3 Theoretically optimal SNCR versus $\alpha(\mathrm{N}=180) \ldots \ldots . . .27$

$4 \quad$ Optimal ratio $s$ versus $\eta \ldots \ldots \ldots$

$5 \quad$ Diagnostic check for the model given by equation (13) . . . . . 29

$6 \quad$ Median efficiencies of sub-optimal designs $(\alpha=0.10) \ldots 34$

$7 \quad$ Median efficiencies of sub-optimal designs $(\alpha=0.50) \ldots 36$

8 Median ratios of sub-optimal designs $(\alpha=0.01) \ldots 36$

$9 \quad$ Median ratios of sub-optimal designs $(\alpha=0.50) \ldots 37$

10 Median optimal SNCR versus $N \ldots \ldots$. . . . . . . 39

11 Dose-response curve for C.I. Acid Red 114 . . . . . . . . . . . 41 


\section{List of Tables}

1 Number of optimal designs derived . . . . . . . . . . . 25

2 Summary of practical optimal SNCR versus. $\alpha(\mathrm{N}=180) \quad$. 27

3 Summary of theoretical optimal SNCR versus. $\alpha(\mathrm{N}=180)$. 28

$4 \quad$ Summary of the four sub-optimal designs used in the study . . 33

$5 \quad$ Reciprocals of median efficiencies $(\alpha=0.10) \ldots \ldots 34$

$6 \quad$ Optimal ratio $s$ at different values of $\eta \ldots \ldots . \ldots 34$

$7 \quad$ Theoretical optimal SNCR versus total sample size $N$. . . . 39

8 Summary of results from observed experiment involving C.I.

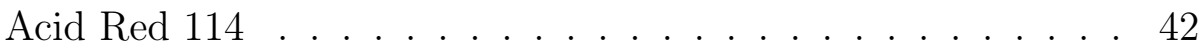

9 Summary of restricted optimal design associated with the results in Table $8 \ldots \ldots \ldots . \ldots . \ldots . \ldots 42$ 


\section{Introduction}

On 11 March 2011, a Mw 9.0 earthquake struck Japan and took more than 15,000 lives. This unfortunately was not the end of tragedy. It caused a devastating tsunami that hit the Fukushima nuclear plants, which resulted in a high level of radiation leakage. Shortly after the incident, the Japanese government announced a temporary increase in the acceptable exposure level for radiation from $1 \mathrm{mSv} /$ year to $20 \mathrm{mSv}$ /year. People were frightened and panicked, as the government failed to deliver a clear justification for their

decision. We then wonder, what is a safe exposure level for radiation? To answer such a question, we must rely on statistical analyses to determine virtually safe levels of exposure to hazardous substances.

\subsection{Derivation of the endpoints for setting human ex- posure guidelines}

The goal of human exposure guidelines is to determine the Reference Dose (RfD) $d$, an estimate of exposure level for the human population including sensitive subgroups, which is free of appreciable risk of deleterious effects during a lifetime. [2]

The derivation of the RfD takes two steps: the determination of the Point of Departure (PoD), and the application of an Uncertainty Factor (UF) to the PoD.

The PoD may be derived from an experiment using methods such as the No Observed Adverse Effect Level (NOAEL) and the Benchmark Dose (BMD). These methods are explained in detail in the following sections. 
Once the PoD is derived, the UF is applied to determine the RfD. Assuming that the estimated PoD is risk-free, a 100-fold UF is applied as a standard UF for carcinogenic experiments employing non-human subjects, while a 10-fold UF can account for animal-to-human variability, and another 10-fold UF is included as a safeguard for sensitive sub-populations.

\subsection{Methods for deriving a point of departure}

In this section, we shall explain two methodologies that are widely used for developing the PoDs used in health risk assessment. The NOAEL method was introduced by Lehman and Fitzhugh in 1954 [8]. The BMD methodology was developed by Crump in 1984 [7].

\subsubsection{The method of NOAEL}

As its name implies, the NOAEL is derived as the highest observed experimental dose level that shows no adverse effect. The NOAEL is based on the assumption that the agent under investigation has a threshold, where any dose under the threshold would not cause adverse effects. The NOAEL becomes difficult to conceptualize when there is a non-zero background risk of developing an adverse effect.

It should also be noted that the NOAEL is constrained to be one of the specified dose levels in a given study, as the method does not assume any mathematical models for the dose-response relationship in its derivation. This may seem to be overly restrictive from a practical perspective, as the NOAEL is not guaranteed to be found in a single particular experiment.

Another criticism that the NOAEL faces is that the likelihood of observing 
an adverse effect increases as the number of tested subjects increases. In other words, an NOAEL derived from smaller and/or poorer designs may provide a greater PoD, introducing a false sense of safety.

Finally, it is also worthwhile noting that the NOAEL may not be risk free. In fact, using 786 experiments from the National Toxicology Program (NTP) database, Sand, Portier, and Krewski (2011) [9] found the median 95\% upper confidence bound on extra risk at the NOAEL to be $11 \%$.

\subsubsection{The method of BMD}

The method of BMD was developed to overcome the shortcomings of the NOAEL. In order to employ the method of BMD, one must first specify the benchmark response (BMR): an acceptable/tolerable level of increase in risk with respect to the prevalence rate in the exposure-free population. The BMR is either given in terms of excess or extra risk. The U.S. Environmental Protection Agency (U.S. EPA) suggests that an excess risk of $10 \%$ be used as the default BMR for quantal experiments, while 5\% BMR may be used for reproductive and developmental studies. For quantal human experiments with greater sensitivities, $1 \%$ excess risk is often employed [1].

In an effort to ensure that the estimated PoD from the BMD method does not exceed the specified BMR, a lower confidence limit (typically 95\%) of the estimated BMD, called the BMDL, is used as the PoD in practice.

There are two major advantages to the BMD method over the NOAEL. One is that the BMD method mathematically evaluates the dose-response relationship, which enables us to calculate the risk at the estimated PoD. 
Another advantage is that, unlike NOAEL, the BMD estimate does not need to be one of the dose levels specified in a study.

However, there are difficulties associated with the method of BMD as well. In 2009, the European Food Safety Authority (EFSA) [3] stated that

Ideally, the BMR would reflect an effect size that is negligible or non-adverse. However, the practical constraint is that the BMR chosen should not be too small, to avoid having to estimate a BMD by extrapolation outside the range of observation, such that the lower confidence limit on the benchmark dose (BMDL) (BMDL) would then depend heavily on the (mathematical) model used.

Given the practical constraints, the determination of an appropriate BMR may sometimes be troublesome. Specifically, in quantal experiments with extremely steep dose-response curve the acceptable level of risk may be much lower than what the practical constraint suggests. (Sand et al. 2011)

\subsubsection{The method of SNCD}

In the hopes of deriving a PoD for the purpose of risk assessment without low-dose extrapolation, Sand et al. (2011) introduced the method of the Signal-to-Noise Crossover Dose (SNCD) using the concept of the Signal-toNoise Ratio (SNR). In what follows, assume that there are $t$ dose groups, where the $i^{\text {th }}$ group has $n_{i}$ subjects. Let $P(d)$ denote the probability of observing an adverse effect at dose level $d$. For the SNCD, the signal at dose 
$d$ is defined to be the additional risk $\mu=\pi(d)=P(d)-P(0)$, and the noise is defined as the width of the $90 \%$ confidence interval about the absolute risk at dose $d\left(P_{95}-P_{05}\right)$. The SNCD is defined as a dose $d^{*}$ such that the ratio is $1\left(\mathrm{SNCD}_{1}\right)$ or $2 / 3\left(\mathrm{SNCD}_{0.67}\right)$. Mathematically,

$$
S N C D_{\omega}=\left\{d: \frac{P(d)-P(0)}{P_{95}-P_{05}}=\omega, 0<d\right\}
$$

where $\omega=1$ or $2 / 3$.

One of the main objectives of the SNCD is to provide a point estimate where the signal can be reliably detected from the noise on a dose-response curve (Sand et al. 2011).

The following summarizes their findings:

- BMDL with associated BMR of $0.01\left(\mathrm{BMDL}_{01}\right)$ and $0.05\left(\mathrm{BMDL}_{05}\right)$ are lower than the SNCD in general.

- The SNCD tends to decrease compared to the NOAEL and the BMDL for datasets with greater information in the low-dose region.

- Using a target extra risk of $1 / 1000$, the median ratios of RfDs based on $\mathrm{BMDL}_{10}, \mathrm{SNCD}_{1}, \mathrm{SNCD}_{0.67}$, and NOAEL are 1.

- The SNCD decreases as the sample size increases; however, the RfD based on SNCD increases as the sample size increases. 


\subsection{The SNCD and its implications}

\subsubsection{The SNR}

For the SNCD, the SNR plays an essential role in its determination of the Virtually Safe Dose (VSD) $d^{*}$. In this section, we shall build a statistical foundation for the SNR used for the SNCD in Sand et al. (2011), where they argued that the SNCD is a point estimate in dose-response curve where the signal can be reliably detected.

In what follows, assume that the $n_{i}$ are sufficiently large so that the estimator of the probability of an adverse effect at any dose level has approximately a normal distribution. Then, the quantity $P_{95}-P_{05}$ in the denominator of the equation (1) can be approximated by $2 z_{0.95} \sigma$, where $\sigma$ is the standard deviation of the estimator of the probability and $z_{0.95}$ is the $95^{\text {th }}$ percentile of the standard normal distribution; that is $z_{0.95}=1.645$. Then the SNR is given by

$$
\begin{aligned}
\omega & =\frac{P(d)-P(0)}{P_{95}-P_{05}} \approx \frac{\mu}{2 z_{0.95} \sigma} \Rightarrow \\
\frac{\mu}{\sigma} & =2 \omega z_{0.95}=3.29 \omega
\end{aligned}
$$

Now suppose that we are interested in testing the following hypotheses for a signal $\mu$ that corresponds to an SNR of $\omega$ :

$$
\begin{aligned}
& H_{0}: \text { signal absent (i.e., } \mu=0 \text { ) } \\
& H_{a}: \text { signal present (i.e., } \mu=\omega \sigma \text { ) }
\end{aligned}
$$

Recall that if a random variable $Y$ is normally distributed with mean 
$\mu$ and variance $\sigma^{2}, X=\left(\frac{Y}{\sigma}\right)^{2}$ would be distributed as non-central $\chi_{1}^{2}$ with non-central parameter $\omega^{2}$. In other words, a random variable $\mathrm{X}$ would be distributed as a central $\chi_{1}^{2}$ under $H_{0}$.

To test the above, we can therefore consider

$$
\left(2 \omega z_{0.95}\right)^{2}=10.82 \omega^{2}
$$

Thus, if we let $\omega=1$ corresponding to $\mathrm{SNCD}_{1}$, we have

$$
x_{1}=10.82
$$

which gives a p-value of $10^{-3}$ under $H_{0}$, which suggests that there is a strong likelihood of the signal's presence.

Similarly, if we let $\omega=2 / 3$ corresponding to $\mathrm{SNCD}_{0.67}$

$$
x_{0.67}=4.81
$$

with a p-value of 0.028 . Thus, in this situation, the null hypothesis would be rejected in favour of the alternate at $5 \%$ type I error rate.

The readers should be cautioned that the above results are based on two assumptions: the confidence bounds are of the excess risk, rather than the absolute risk; and the given confidence bounds are based on the normality assumption, rather than derived by the profile likelihood method (its derivation is given in Appendix A.4).

\subsubsection{Twisting the concept of SNCD}

Although one may argue that it is reasonable to believe that additional risks at $\mathrm{SNCD}_{1}$ and $\mathrm{SNCD}_{0.67}$ can be reliably detected, there is some ambiguity in the definition of the SNCD. First, the noise level used is of the 
absolute risk, while the signal is defined in terms of the excess risk. Second, the use of the width of the $90 \%$ confidence interval $P_{95}-P_{05}$ (or $2 / 3$ of it) as the definition of background noise is rather arbitrary from a statistical point of view.

Thus we propose a new SNR to be used for determining the SNCD, which is defined as:

$$
S N R: \frac{\mu}{\sigma}=\frac{\pi(d)}{\sigma_{\hat{\pi}(d)}}=\lambda
$$

where $\pi(d)$ is the true excess risk at dose $d$, and $\sigma_{\hat{\pi}(d)}$ denotes the standard error given by the maximum likelihood estimator (MLE) $\hat{\pi}(d)$. The quantity $\lambda$ is chosen to be such that the signal is relatively small compared to the noise that we cannot detect the signal with enough certainty. In particular, we choose $\lambda=\chi_{1,0.9}$, as this implies that the signal can be detected from the noise with $90 \%$ confidence, which is the minimal level of confidence used to reject the null hypothesis presented in section 1.3.1.

Therefore, the SNCD is redefined to be:

$$
S N C D=\left\{d: \frac{\pi(d)}{\sigma_{\hat{\pi}(d)}}=\chi_{1,0.9}, 0<d\right\}
$$

Note that the SNR is based on a chi-distribution, rather than on a chisquared distribution.

Throughout the paper, SNCD is based on equation (3), and we refer to the excess risk associated with the SNCD as the Signal-to-Noise Crossover Risk (SNCR). Note that the original SNCD defined by Sand et al. will be referred as $\mathrm{SNCD}_{\text {orig }}, \mathrm{SNCD}_{1}$, or $\mathrm{SNCD}_{0.67}$.

The goal of this new definition is to employ SNCR as a measure of an 
increase in risk to be used in the determination of $\mathrm{PoD}$ and RfD that is ground in statistical theory.

\subsection{The objectives of this study}

One of the main goals of this paper is to build the theoretical foundations for the SNCD and SNCR with a view towards investigating the optimal design. Specifically, once the theoretical foundations are formulated, the feasibility of the optimal design for SNCR is examined under different doseresponse relationships as described by appropriately chosen parameters in a Hill model. A comparison of SNCR based on the optimal design to SNCR based on a sub-optimal design is also included. An illustrative example based on the National Toxicology Program (NTP) carcinogenesis database of experiments is also presented.

\subsection{The Hill model}

The Hill model is a way to describe epidemiological dose-response relationships. It is particularly useful when the background rate (i.e., the probability of an adverse event at a dose level of zero) is non-zero. Throughout the paper, we are assuming that the risk for the exposure-free population is nonzero and independent of the effect of the agent under investigation. Under such an assumption the three-parameter Hill model is defined as follows:

$$
P(d \mid \boldsymbol{\theta})=P(d \mid \alpha, \kappa, \eta)=\alpha+(1-\alpha) \frac{d^{\eta}}{d^{\eta}+\kappa^{\eta}}
$$

where $\alpha$ represents the level of the background response rate; $\kappa$ is the effective dose of $50\left(\mathrm{ED}_{50}\right)$; and $\eta$, the Hill coefficient, describes the shape of the doseresponse curve. 
One of the assets that the Hill model provides compared to models such as the Weibull and one-hit models, is the ease in interpretation of its parameters.

The parameter spaces for the Hill model parameters are given as follows:

Under the assumption of non-zero background, $\alpha$ is constrained to be in $(0,1)$. The only constraint that $\kappa$ faces is that it must be positive. Finally, since we are interested in modelling a monotonically increasing dose-response trend, $\eta$ theoretically could be any positive value. However, in order to avoid supra-linearity in the low dose region, in our study here we constrain $\eta$ to be at least one.

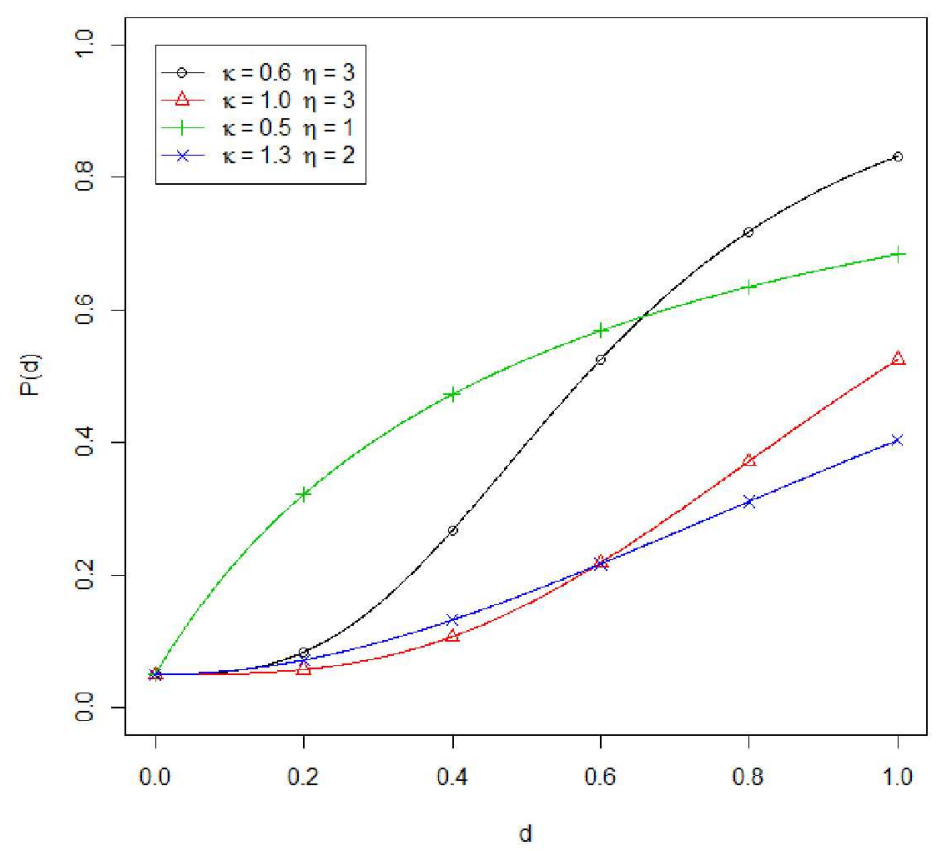

Figure 1: Four types of dose-response relationship under the Hill model 


\subsection{Four types of dose-response relationships}

Using the three-parameter Hill model, we can classify the observed doseresponse curves into four categories: sigmoidal, near-linear, concave, and convex. Figure 1 gives illustrative examples of the four types of dose-response relationships with $\alpha$ of 0.05 by varying $\kappa$ and $\eta$.

The concave curve is characterized by the Hill parameter $\eta$ being one. When $\eta$ is greater than one, an experiment may be described by any of the sigmoidal, convex, or near-linear dose-response curves.

In order to observe a sigmoidal dose-response relationship in the experimental range, the Maximum Dose Tested (MDT) needs to be large enough to capture both the lower and higher inflection points. In other words, $\kappa$ needs to be relatively (but not too) small compared to the MDT. In particular, $\kappa$ should be somewhere in between $20 \%$ to $80 \%$ of the MDT. It's also worthwhile noting that $\eta$ should not be too large, as it may result in an unreasonably steep curve, which almost acts like a step function.

Note that convexity and near-linearity of the observed dose-response relationship are mostly due to relatively small MDT compared to $\kappa$. In Figure 1, we observe that for both sigmoidal and convex curves, the value of $\eta$ is 3 ; the only difference is $\kappa$. In other words, the concavity is simply a result given by setting the MDT to be less than $\kappa$. If $\kappa$ is much greater, we would only be able to capture a near-linear relationship in the experimental range. 


\section{Developing Optimal Designs for SNCR}

The derivation of the optimal design for SNCR is an extension of the optimal design for BMD and corresponding BMR (see section 2.3). Therefore we discuss the method to obtain the optimal design for BMD prior to the discussion of the optimal design for SNCR. Note that the results in this section mainly follow the work of Krewski, Kover, and Bickis (1984) [5].

\subsection{MLEs and Optimal Design for BMD/BMR}

\subsubsection{Derivation of the MLE and covariance matrix for the Hill model parameters}

Suppose that we have $t$ dose levels to test with a total sample size of $N$; each dose group has $n_{i}$ test subjects. Let $Y_{i j}$ be a response from the $j^{\text {th }}$ subject in the $i^{\text {th }}$ dose group, which may either be 0 (response absent) or 1 (response present), and let $P\left(d_{i}\right)$ denote the probability of observing an adverse effect on a test subject from the $i^{\text {th }}$ dose group. Then we have

$$
Y_{i j} \sim \text { Bernoulli }\left[P\left(d_{i}\right)\right]
$$

Furthermore, assuming that the response of each subject is independent of the others at a given dose level, we have

$$
X_{i}=\sum_{j=1}^{n_{i}} Y_{i j} \sim \operatorname{Bin}\left[n_{i}, P\left(d_{i}\right)\right]
$$

Assuming that the probability at dose $d_{i}$ follows the Hill model with completely independent background as given in equation (4) and setting $\boldsymbol{\theta}=$ 
$(\alpha, \kappa, \eta)=\left(\theta_{1}, \theta_{2}, \theta_{3}\right)$, we have

$$
P\left(d_{i}\right)=P\left(d_{i} \mid \boldsymbol{\theta}\right)=\alpha+(1-\alpha)\left(\frac{d_{i}^{\eta}}{\kappa^{\eta}+d_{i}^{\eta}}\right)
$$

and the likelihood is given by

$$
L(\boldsymbol{\theta})=f(\boldsymbol{x} \mid \boldsymbol{\theta})=\prod_{i=1}^{t}\left(\begin{array}{c}
n_{i} \\
x_{i}
\end{array}\right)\left[P\left(d_{i}\right)\right]^{x_{i}}\left[1-P\left(d_{i}\right)\right]^{n_{i}-x_{i}}
$$

The log-likelihood is then

$$
\begin{aligned}
l(\boldsymbol{\theta}) & =\ln [L(\boldsymbol{\theta})] \\
& =\sum_{i=1}^{t}\left\{x_{i} \ln \left[P\left(d_{i}\right)\right]+\left(n_{i}-x_{i}\right) \ln \left[1-P\left(d_{i}\right)\right]\right\}+\text { constant }
\end{aligned}
$$

The MLE $\hat{\boldsymbol{\theta}}_{M L E}$ of $\boldsymbol{\theta}$ is obtained by maximizing the likelihood function or log-likelihood function given by equations (5) and (6). Since it is impossible to solve for $\hat{\boldsymbol{\theta}}_{M L E}$ analytically, numerical methods such as the NewtonRaphson method (see Appendix A.1 for details) are required to obtain the MLE.

The covariance matrix associated with $\hat{\boldsymbol{\theta}}_{M L E}$ can be determined by evaluating the inverse of the information matrix $\boldsymbol{X}_{(\hat{\boldsymbol{\theta}})}$. Note that the first and second order partial derivatives of the log-likelihood with respect to each Hill model parameter can be expressed as:

$$
\begin{aligned}
\frac{l(\boldsymbol{\theta})}{\partial\left(\theta_{r}\right)} & =\frac{\partial}{\partial\left(\theta_{r}\right)}\left[\sum_{i=1}^{t}\left\{x_{i} \ln \left[P\left(d_{i}\right)\right]+\left(n_{i}-x_{i}\right) \ln \left[1-P\left(d_{i}\right)\right]\right\}\right] \\
& =\sum_{i=1}^{t}\left\{\frac{P_{r}\left(d_{i}\right)\left[x_{i}-n_{i} P\left(d_{i}\right)\right]}{P\left(d_{i}\right)\left[1-P\left(d_{i}\right)\right]}\right\}
\end{aligned}
$$

where

$$
P_{r}\left(d_{i}\right)=\frac{\partial P\left(d_{i}\right)}{\partial \theta_{r}} ; r=1,2,3
$$


and

$\frac{\partial^{2} l(\boldsymbol{\theta})}{\partial\left(\theta_{r}\right) \partial\left(\theta_{s}\right)}=\sum_{i=1}^{t}\left\{\frac{-n_{i} P_{r}\left(d_{i}\right) P_{s}\left(d_{i}\right)+P_{r, s}\left(d_{i}\right)\left[x_{i}-n_{i} P\left(d_{i}\right)\right]}{P\left(d_{i}\right)\left[1-P\left(d_{i}\right)\right]}+\frac{P_{s}\left(d_{i}\right)\left[x_{i}-n_{i} P\left(d_{i}\right)\right]}{P_{r}\left(d_{i}\right)}\right\}$

with $r, s=1,2,3$

Now, note that the expected number of responses for the $i^{\text {th }}$ dose group is

$$
E\left(X_{i}\right)=n_{i} P\left(d_{i}\right)
$$

Hence the information matrix is given by

$$
\boldsymbol{X}_{(\hat{\boldsymbol{\theta}})}=-E\left[\frac{\partial^{2} l(\boldsymbol{\theta})}{\partial\left(\theta_{r}\right) \partial\left(\theta_{s}\right)}\right]=\sum_{i=1}^{t} \frac{n_{i} P_{r}\left(d_{i}\right) P_{s}\left(d_{i}\right)}{P\left(d_{i}\right)\left[1-P\left(d_{i}\right)\right]}=\boldsymbol{A} \boldsymbol{D} \boldsymbol{A}^{T}
$$

where

$$
\begin{aligned}
& \underset{3 \times t}{\boldsymbol{A}}= {\left[\begin{array}{llll}
P_{1}\left(d_{1}\right) & P_{1}\left(d_{2}\right) & \cdots & P_{1}\left(d_{t}\right) \\
P_{2}\left(d_{1}\right) & P_{2}\left(d_{2}\right) & \cdots & P_{2}\left(d_{t}\right) \\
P_{3}\left(d_{1}\right) & P_{3}\left(d_{2}\right) & \cdots & P_{3}\left(d_{t}\right)
\end{array}\right] \text { and } } \\
& \underset{t \times t}{\boldsymbol{D}}=\operatorname{diag}\left\{\frac{n_{i}}{p_{i} q_{i}}\right\} ; p_{i}=P\left(d_{i}\right), q_{i}=1-p_{i}
\end{aligned}
$$

Hence, $\hat{\boldsymbol{\theta}}_{M L E}$ has covariance matrix $\boldsymbol{\Sigma}_{\hat{\boldsymbol{\theta}}_{M L E}}=\boldsymbol{X}_{(\hat{\boldsymbol{\theta}})}^{-1}$.

\subsubsection{MLEs of BMR and BMD}

Krewski \& Ryzin (1981) [6] showed that, under some regularity conditions (see Appendix A.3 for the proofs), the asymptotic variance of the estimated excess risk over the background risk at $d_{0}: \hat{\pi}_{0}=\hat{\pi}\left(d_{0}\right)$ can be approximated by

$$
V\left(\hat{\pi}_{0}\right)=\boldsymbol{\Pi}^{T} \boldsymbol{X}_{(\hat{\boldsymbol{\theta}})}^{-1} \boldsymbol{\Pi}
$$

where $\Pi^{T}=\left(\Pi_{1}, \ldots, \Pi_{k}\right)$ with $\Pi_{r}(d)=P_{r}(d)-P_{r}(0)$. 
Now, note that for a pre-specified $B M R: \pi_{0}>0$, we get

$$
d_{0}=\frac{\kappa}{\sqrt[\eta]{\frac{1-\alpha}{\pi_{0}}-1}}
$$

Note that if $\eta$ is a multiple of $2, d_{0}$ in equation (7) may have positive and negative solutions. Since we are interested in the dose response model for $d>0$, we will only focus on the positive case.

Furthermore, using the Delta-method, the asymptotic variance of $\hat{d}_{0}$ is given by

where

$$
V\left(\hat{d}_{0}\right)=\left[\frac{\partial P(d)}{\partial d}\right]_{d=\hat{d}_{0}}^{-2} V\left(\hat{\pi}_{0}\right)
$$

That is, we have

$$
\frac{\partial P(d)}{\partial d}=(1-\alpha) \frac{\eta \kappa^{\eta} d^{\eta-1}}{\left(\kappa^{\eta}+d^{\eta}\right)^{2}}
$$

$$
V\left(\hat{d}_{0}\right)=\left[(1-\alpha) \frac{\eta \kappa^{\eta} d_{0}^{\eta-1}}{\left(\kappa^{\eta}+d_{0}^{\eta}\right)^{2}}\right]^{-2} \boldsymbol{\Pi}^{T} \boldsymbol{X}_{(\hat{\boldsymbol{\theta}})}^{-1} \boldsymbol{\Pi}
$$

Under the BMD method, we have

$$
\begin{aligned}
& \hat{d}_{0}=\pi^{-1}\left(\pi_{0} \mid \hat{\boldsymbol{\theta}}\right) \Rightarrow \\
& d_{0}=E\left(\hat{d}_{0}\right)=E\left[\pi^{-1}\left(\pi_{0} \mid \hat{\boldsymbol{\theta}}\right)\right]=\pi^{-1}\left(\pi_{0} \mid \boldsymbol{\theta}\right)
\end{aligned}
$$

Hence (9) is constant under a given $\boldsymbol{\theta}$. This implies that minimizing the variance of $\hat{\pi}_{0}$ is equivalent to minimizing the variance of $\hat{d}_{0}$, suggesting that an optimal design for BMR is also an optimal design for BMD.

\subsubsection{Optimal design for BMD and BMR}

We are interested in estimating the excess risk $\pi_{0}$, which depends on the Hill model parameters $\boldsymbol{\theta}$. 
The Chernoff's theorem (Chernoff, 1952 [4]) states that if the information matrix $\boldsymbol{X}_{(\hat{\boldsymbol{\theta}})}$ is closed and bounded at any $d \in[0, D]$ under any given set of $\boldsymbol{\theta} \in \boldsymbol{\Theta}$, then there exists a set of dose levels $\boldsymbol{d}$, which minimizes the variance of the estimator $\hat{\pi}_{0}$. Furthermore, the theorem tells us that such an optimal design would require three dose levels in an experiment. (See Appendix A.2 for proofs.)

For experiments with three dose levels, we get

$$
\boldsymbol{X}_{(\boldsymbol{\theta})}^{-1}=\left(\boldsymbol{A} \boldsymbol{D} \boldsymbol{A}^{T}\right)^{-1}=\left(\boldsymbol{A}^{T}\right)^{-1} \boldsymbol{D}^{-1} \boldsymbol{A}^{-1}
$$

where

$$
\begin{aligned}
& \underset{3 \times 3}{\boldsymbol{A}^{-1}}=\left\{a^{i r}\right\} \text { and } \\
& \underset{3 \times 3}{\boldsymbol{D}^{-1}}=\operatorname{diag}\left\{\frac{p_{i} q_{i}}{n_{i}}\right\}
\end{aligned}
$$

Hence,

$$
\boldsymbol{X}_{(\boldsymbol{\theta})}^{-1}=\left[\begin{array}{llll}
\sum_{i=1}^{3} a^{i 1} a^{i 1} \frac{p_{i} q_{i}}{n_{i}} & \sum_{i=1}^{3} a^{i 2} a^{i 1} \frac{p_{i} q_{i}}{n_{i}} & \sum_{i=1}^{3} a^{i 3} a^{i 1} \frac{p_{i} q_{i}}{n_{i}} \\
\sum_{i=1}^{3} a^{i 1} a^{i 2} \frac{p_{i} q_{i}}{n_{i}} & \sum_{i=1}^{3} a^{i 2} a^{i 2} \frac{p_{i} q_{i}}{n_{i}} & \sum_{i=1}^{3} a^{i 3} a^{i 2} \frac{p_{i} q_{i}}{n_{i}} \\
\sum_{i=1}^{3} a^{i 1} a^{i 3} \frac{p_{i} q_{i}}{n_{i}} & \sum_{i=1}^{3} a^{i 2} a^{i 3} \frac{p_{i} q_{i}}{n_{i}} & \sum_{i=1}^{3} a^{i 3} a^{i 3} \frac{p_{i} q_{i}}{n_{i}}
\end{array}\right]
$$

which leads to 


$$
\begin{aligned}
V\left(\hat{\pi}_{0}\right) & =\Pi^{T} \boldsymbol{X}_{(\boldsymbol{\theta})}^{-1} \boldsymbol{\Pi} \\
& =\left[\begin{array}{lll}
\Pi_{1} & \Pi_{2} & \Pi_{3}
\end{array}\right] \boldsymbol{X}_{(\boldsymbol{\theta})}^{-1}\left[\begin{array}{l}
\Pi_{1} \\
\Pi_{2} \\
\Pi_{3}
\end{array}\right] \\
& =\left[\begin{array}{l}
\left.\sum_{i=1}^{3} \frac{p_{i} q_{i}}{n_{i}} \sum_{j=1}^{3} \Pi_{j} a^{i 1} a^{i j}, \cdots, \sum_{i=1}^{3} \frac{p_{i} q_{i}}{n_{i}} \sum_{j=1}^{3} \Pi_{j} a^{i 3} a^{i j}\right] \\
\Pi_{2} \\
\Pi_{2} \\
\Pi_{3}
\end{array}\right] \\
& =\sum_{i=1}^{3} \frac{p_{i} q_{i}}{n_{i}} \sum_{j=1}^{3} \Pi_{j} a^{i j} \Pi_{1} a^{i 1}+\cdots+\sum_{i=1}^{3} \frac{p_{i} q_{i}}{n_{i}} \sum_{j=1}^{3} \Pi_{j} a^{i j} \Pi_{3} a^{i 3} \\
& =\sum_{i=1}^{3} \frac{p_{i} q_{i}}{n_{i}} \sum_{j=1}^{3} \Pi_{j} a^{i j}\left(\Pi_{1} a^{i 1}+\cdots+\Pi_{3} a^{i 3}\right) \\
& =\sum_{i=1}^{3} \frac{p_{i} q_{i}}{n_{i}}\left(\sum_{j=1}^{3} \Pi_{j} a^{i j}\right)^{2}
\end{aligned}
$$

Now, we shift our attention to minimizing the variance at given $\hat{\pi}_{0}$ and $\boldsymbol{d}=\left(d_{1}, d_{2}, d_{3}\right)$. Let

$$
\begin{aligned}
Q & =V\left[\hat{\pi}_{0}\right] \\
& =V\left[\hat{\pi}_{0}\right]+\phi\left(N-\sum_{i=1}^{3} n_{i}\right) \\
& =\sum_{i=1}^{3} \frac{p_{i} q_{i}}{n_{i}}\left(\sum_{j=1}^{3} \Pi_{j} a^{i j}\right)^{2}+\phi\left(N-\sum_{i=1}^{3} n_{i}\right)
\end{aligned}
$$

Taking the partial derivatives with respect to $n_{i}$ and $\phi$ yield

$$
\frac{\partial Q}{\partial n_{i}}=\frac{p_{i} q_{i}}{n_{i}^{2}}\left(\sum_{j=1}^{3} \Pi_{j} a^{i j}\right)^{2}+\phi
$$


and

$$
\frac{\partial Q}{\partial \phi}=\left(N-\sum_{i=1}^{3} n_{i}\right)
$$

Then, setting (11) equal to 0 , we obtain

$$
n_{i}=\sqrt{\frac{p_{i} q_{i}\left(\sum_{j=1}^{3} \Pi_{j} a^{i j}\right)^{2}}{\phi}}
$$

and since

$$
N=\sum_{i=1}^{3} n_{i}=\sum_{i=1}^{3} \sqrt{\frac{p_{i} q_{i}\left(\sum_{j=1}^{3} \Pi_{j} a^{i j}\right)^{2}}{\phi}}
$$

we obtain asymptotically optimal allocations of proportion $\boldsymbol{c}=\left(c_{1}, c_{2}, c_{3}\right)$ for each dose level:

$$
c_{i}=\frac{n_{i}}{N}=\frac{\sqrt{\frac{p_{i} q_{i}\left(\sum_{j=1}^{3} \Pi_{j} a^{i j}\right)^{2}}{\phi}}}{\sum_{i=1}^{3} \sqrt{\frac{p_{i} q_{i}\left(\sum_{j=1}^{3} \Pi_{j} a^{i j}\right)^{2}}{\phi}}}=\frac{\sqrt{p_{i} q_{i}}\left|\sum_{j=1}^{3} \Pi_{j} a^{i j}\right|}{\sum_{i=1}^{3}\left\{\sqrt{p_{i} q_{i}}\left|\sum_{j=1}^{3} \Pi_{j} a^{i j}\right|\right\}}
$$

A numerical search on the triplets $\boldsymbol{d}=\left(d_{1}, d_{2}, d_{3}\right)$ is then executed to find the optimal dose levels that minimizes $V\left(\hat{\pi}_{0}\right)$.

Hence, substituting (12) into (10), we obtain the variance of $\hat{\pi}_{0}$ under the optimal design, which is

$$
V\left(\hat{\pi}_{0}\right)=\left(\sum_{i=1}^{3} p_{i} q_{i}\left|\sum_{j=1}^{3} a^{i j} \pi_{j}\left(\hat{d}_{0}\right)\right|\right)^{2}
$$

\subsection{Search of an explicit solution for SNCD from a given experiment}

The BMD depends only on the Hill parameters $\boldsymbol{\theta}$ and the pre-determined BMR: it can be solved explicitly as given in equation (7). On the other 
hand, the SNCD depends on $V\left(\hat{\pi}_{0}\right)$, which is not only a function of the Hill model parameters $\boldsymbol{\theta}$, but also of the experimental design. From an observed experiment, we wish to solve the SNCD explicitly so that we can find an optimal design under some optimality criteria.

The SNCD must satisfy the SNR given in equation (2). By re-expressing the SNR, we get

$$
\begin{aligned}
\pi\left(d_{0} \mid \boldsymbol{\theta}\right) & =\lambda \sigma_{\hat{\pi}\left(d_{0} \mid \boldsymbol{\theta}\right)} \Rightarrow \\
\pi\left(d_{0} \mid \boldsymbol{\theta}\right) & =\lambda\left[\boldsymbol{\Pi}^{T} \boldsymbol{X}_{(\hat{\boldsymbol{\theta}})}^{-1} \boldsymbol{\Pi}\right]^{1 / 2}
\end{aligned}
$$

The argument based on the Chernoff's theorem for the derivation of the optimal design for BMR also works for the optimal design for SNCR as both BMR and SNCR are expressed in terms of excess risks. Hence, if we focus our attention to what happens in experiments with three dose levels, we get

$$
\pi\left(d_{0} \mid \boldsymbol{\theta}\right)=\lambda\left[\sum_{i=1}^{3} \frac{p_{i} q_{i}}{n_{i}}\left(\sum_{j=1}^{3} \Pi_{j}\left(d_{0}\right) a^{i j}\right)^{2}\right]^{1 / 2}
$$

In order to explicitly solve for SNCD and SNCR, we must isolate $\pi\left(d_{0} \mid \boldsymbol{\theta}\right)$ and $\Pi_{j}\left(d_{0}\right), j=1,2,3$ on one side; however, this is impossible. Hence, the search for the values of SNCD and SNCR from a given experiment must be derived through a numerical analysis.

\subsection{Optimal designs for SNCD and SNCR}

As defined in (3), the SNCD is a dose level that satisfies a certain SNR, $\lambda$, for a given sample size $N$ and Hill model parameters $\boldsymbol{\theta}$ (or their estimates $\hat{\boldsymbol{\theta}}$.) Suppose that we are interested in deriving optimal designs for SNCD and SNCR such that the asymptotic variances of SNCD and/or SNCR are 
minimized.

In section 2.1.3, we showed that minimizing the variance of BMR was equivalent to minimizing the variance of the corresponding BMD. Unfortunately, minimizing the variance of SNCR does not necessarily result in the optimization of the variance of the corresponding SNCD.

Suppose that we are interested in minimizing the variance of the SNCR estimate. This means that the optimal design for SNCR yields a locally minimized estimate of the SNCR.

From (8), we know that the variance of the SNCD corresponding to an estimated SNCR is given by:

$$
V(\widehat{S N C D})=\left(\left.\frac{\partial P(d)}{\partial d}\right|_{d=\widehat{S N C D}}\right)^{-2} V(\widehat{S N C R})
$$

When $\eta=1, \frac{\partial P(d)}{\partial d}$ increases as $d$ approaches 0 . Hence minimizing the variance of an SNCR estimate is equivalent to minimizing the variance of an estimated SNCD. However, if $\eta>1, \frac{\partial P(d)}{\partial d}$ decreases as $d$ approaches 0 . Therefore, minimizing the variance of an estimated SNCR does not necessarily result in minimizing the variance of the corresponding SNCD estimate. In fact, as the growth of $\left(\frac{\partial P(d)}{\partial d}\right)^{-2}$ is much greater than the reduction in $V(\hat{d})$ in the low dose region, often times the variance of the SNCD estimate is not optimized by minimizing the variance at an estimated SNCR.

In this paper, we propose to focus on the optimal design for SNCR. Recall that our intention of employing the new definition for the signal-to-noise cross over dose is to determine the minimum additional risk that can reject the null 
hypothesis, for a given sample size $N$ and Hill model parameters $\boldsymbol{\theta}$, namely $H_{0}$ : Increase in risk at SNCD is zero

Hence, the optimal design for SNCR ensures us that any dose less than SNCD would not result in a statistically significant increase in risk.

On the other hand, minimizing the variance at SNCD implies that, unless $\eta=1$, it is likely that the design gives a dose level that corresponds to an additional risk that satisfies the SNR under non-minimal variance. This gives a rather arbitrary dose that does not coincide with the concept of PoD. Hence throughout this study we use the optimal design for SNCR rather than the one for SNCD.

\subsection{Optimal Design for SNCR}

As discussed in the previous section, the SNCR and SNCD depend on the Hill model parameters, as well as the experimental design. Hence the optimal design for SNCR must be obtained using an iterative method, which is given as follows:

Step 1: Obtain an optimal design for some target risk, say of 10\% excess risk, and estimate the SNCR under $\mathrm{OD}_{B M R_{10}}$. Denote the estimated SNCR by $\widehat{S N C R}^{(1)}$.

Step 2: Set the target risk to be $\widehat{S N C R}^{(1)}$ and obtain an optimal design and re-estimate the SNCR under $\mathrm{OD}_{S N C R^{(1)}}$. Denote the SNCR by $\widehat{S N C R}^{(2)}$.

Step 3: Repeat Step 2 until $\left|\widehat{S N C R}^{(m)}-\widehat{S N C R}^{(m-1)}\right|<10^{-8}$. This gives an optimal design for given $\boldsymbol{\theta}$ and $N$. 


\section{Feasibility Study for Optimal Designs}

In the previous section, we have built some of the theoretical foundation underlying the SNCD and the optimal design for the SNCR. In this section, we investigate the feasibility of obtaining an optimal design for a variety of dose-response relationships. Specifically, we shall investigate the effects of the Hill model parameters, the total sample size, and the experimental design.

\subsection{Parameters used in the study}

While we set the Maximum Tolerated Dose (MTD) to be one, and the total sample size to be 180 , we consider the following ranges for the Hill model parameters:

$$
\begin{aligned}
\alpha & =0.0001,0.001,0.01,0.05,0.1,0.2,0.3,0.4,0.5,0.6 \\
\kappa & =0.1,0.2, \cdots, 2.0 \\
\eta & =1,2, \cdots, 10
\end{aligned}
$$

We now justify these choices. Since the cases of non-zero background are of interest, we consider values of $\alpha$ that exceed zero. However, as illustrated in Table 1 , when $\alpha$ is less than 0.01 , the derivation of a practical optimal design is virtually non-existent, as the allocation scheme for the control group is so small that the optimal design would only involve two dose levels. The choice of the maximum value for $\alpha(0.6)$ is motivated by the carcinogenesis experiments abstracted from the NTP database

When $\kappa$ is outside of the experimental dose range (i.e., $\kappa>1$ ), the derivation of practical optimal designs is nearly impossible. Therefore we constrain 
ourselves to studying only the cases where $\kappa$ is at most twice as large as the MTD.

In order to avoid supra-linearity in the low-dose region of the doseresponse curve, the minimum $\eta$ for this study is set to one. The maximum value of 10 selected above is based on Sand et al (2011), since an $\eta$ above this value would result in an unreasonably steep dose-response relationship.

The total sample size of 180 is used for this study in order to ensure realistic sample allocations in the sub-optimal designs described in section 3.5.

At each background rate $\alpha$ considered, 200 different sets of $\kappa$ and $\eta$ are employed, which give rise to a total of 2000 different sets of the Hill model parameters.

\subsection{Process of obtaining optimal designs for SNCR}

For each of these sets, a numerical search for the optimal design for $\mathrm{BMD}_{10}$ is first conducted. Since the standard practice requires the control group to be included in an experiment, the lowest experimental dose level is set to zero. Furthermore, we restrict the MDT to not exceed the MTD, as an extremely large dose level may distort the biological attribute of the doseresponse relationship. Given these constraints, the optimal maximum- and intermediate dose levels are determined through a two-dimensional 1/100th

grid search. For each set of experimental dose levels $\boldsymbol{d}$, an optimal allocation of proportions $\boldsymbol{c}$ is calculated, and the design $(\boldsymbol{d}, \boldsymbol{c})$ with minimum variance at BMD is determined. 
In order to validate the practicality of the optimal schemes, the potential optimal designs must satisfy the following to be considered for the consequent analyses:

1) No pair of dose levels must be adjacent to each other; that is, as a result of the above grid search, the intermediate dose level $d_{2}$ must be greater than 0.01 and at least two grids less than $d_{3}$.

2) Each of the proportions of allocation $c_{i}$ must be greater than $5 \%$, as a lower allocation would not be practical.

Once the optimal designs for $\mathrm{BMD}_{10}$ are derived and practical criteria are validated, the optimal designs for SNCR are searched. Again, in order for the SNCR optimal designs to be considered for the study, they must satisfy the practicality criteria described above.

\section{$3.3 \quad$ Results}

Table 1 summarizes the number of optimal designs derived for the $\mathrm{BMD}_{10}$ and the SNCR. Note that $c_{1}, c_{2}, c_{3}$ represent the number of designs that did not satisfy $c_{i}>0.05, i=1,2,3$, while $d_{2} d_{1}$ and $d_{3} d_{2}$ denote the number of designs where the intermediate optimal dose was adjacent to 0 or MDT, respectively.

From Table 1, we can draw a few observations. As mentioned earlier, at $\alpha=0.0001$, the risk at the control group is so small that the allocation scheme for $d_{1}$ falls under $5 \%$ in almost all situations. This does not seem to 


\begin{tabular}{|c|c|c|c|c|c|c|c|c|c|c|}
\hline$\alpha$ & $\begin{array}{c}\text { Number of } \\
\text { Experimental Designs }\end{array}$ & $\begin{array}{c}\text { Number of } \\
\mathrm{OD}_{B M D_{10}} \text { derived }\end{array}$ & $c_{1}$ & $c_{2}$ & $c_{3}$ & $d_{2} d_{1}$ & $d_{3} d_{2}$ & $\begin{array}{l}\text { Number of practical } \\
\mathrm{OD}_{B M D_{10}} \text { derived }\end{array}$ & $\begin{array}{l}\text { Number of practical } \\
\mathrm{OD}_{S N C R} \text { derived }\end{array}$ & $\begin{array}{l}\text { Number of theoretical } \\
\text { OD }_{S N C R} \text { derived }\end{array}$ \\
\hline 0.0001 & 200 & 148 & 144 & 4 & 18 & 1 & 126 & 4 & 0 & 0 \\
\hline 0.001 & 200 & 148 & 8 & 3 & 49 & 1 & 94 & 9 & 9 & 2 \\
\hline 0.01 & 200 & 148 & 8 & 4 & 28 & 0 & 62 & 48 & 48 & 37 \\
\hline 0.1 & 200 & 148 & 8 & 14 & 20 & 0 & 29 & 68 & 64 & 29 \\
\hline 0.2 & 200 & 148 & 10 & 4 & 20 & 0 & 35 & 72 & 64 & 25 \\
\hline 0.3 & 200 & 148 & 4 & 2 & 23 & 1 & 34 & 74 & 74 & 38 \\
\hline 0.4 & 200 & 147 & 12 & 13 & 20 & 0 & 30 & 69 & 67 & 37 \\
\hline
\end{tabular}

Table 1: Number of optimal designs derived 
be an issue for $\alpha$ greater than or equal to 0.001 . When $\alpha$ equals $10^{-4}$ or $10^{-3}$, the intermediate dose and the maximum dose tested (MDT) are frequently adjacent to each other, implying that designs with a negligible background rate are essentially two-dose experiments.

In general, the optimal intermediate dose would not be adjacent to the control group. The optimal $d_{2}$ may practically be zero only if $\kappa$ is set so small with respect to MDT, making the searched grids too coarse.

By analyzing the practical optimal designs, we find that the SNCR is largely dependent on the $\alpha$ estimate. Figure 2 shows a box-plot of SNCR against varying $\alpha$ values for the various combinations of $\kappa$ and $\eta$. Summary statistics for SNCR are given in Table 2.

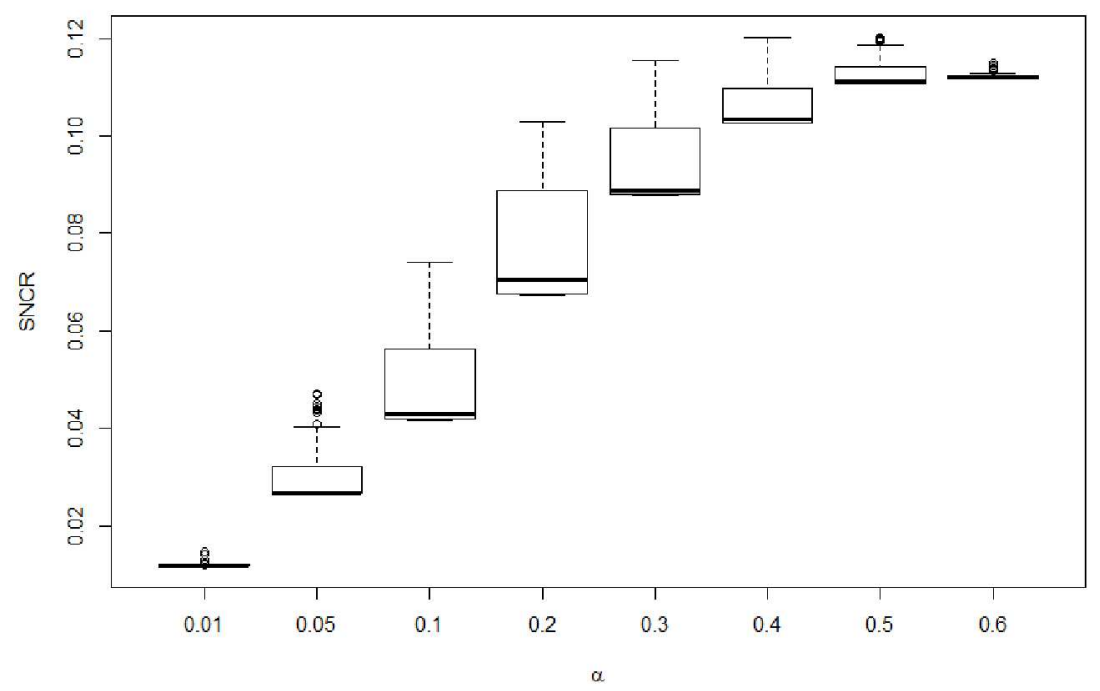

Figure 2: Optimal SNCR versus $\alpha(\mathrm{N}=180)$

Figure 2 shows that the distribution of SNCRs are heavily skewed to the right at each level of $\alpha$ considered. This is primarily due to the fact 


\begin{tabular}{|l|l|l|l|l|l|l|l|l|}
\hline $\boldsymbol{\alpha}$ & 0.01 & 0.050 & 0.1 & 0.2 & 0.3 & 0.4 & 0.5 & 0.6 \\
\hline min & 0.0118 & 0.0266 & 0.0417 & 0.0674 & 0.0878 & 0.1025 & 0.1108 & 0.1118 \\
\hline mean & 0.0121 & 0.0305 & 0.0494 & 0.0779 & 0.0949 & 0.1066 & 0.1130 & 0.1124 \\
\hline median & 0.0118 & 0.0268 & 0.0428 & 0.0704 & 0.0888 & 0.1033 & 0.1113 & 0.1120 \\
\hline max & 0.0148 & 0.0472 & 0.0742 & 0.1029 & 0.1154 & 0.1202 & 0.1201 & 0.1150 \\
\hline
\end{tabular}

Table 2: Summary of practical optimal SNCR versus. $\alpha(\mathrm{N}=180)$

that MTD is set to one. For some values of $\kappa$ and $\eta$, the MTD of one is not large enough to yield a theoretical optimal design, leading to it being a restricted optimal design with a relatively larger SNCR. It should also be noted that the optimal design would only require two dose levels when $\eta$ is one. Thus, if we consider box-plots of SNCR for values of $\kappa$ and $\eta$ that only yield theoretical optimal designs (i.e., experiments where the MDT is less than one), we observe the following results in Figure 3 and Table 3.

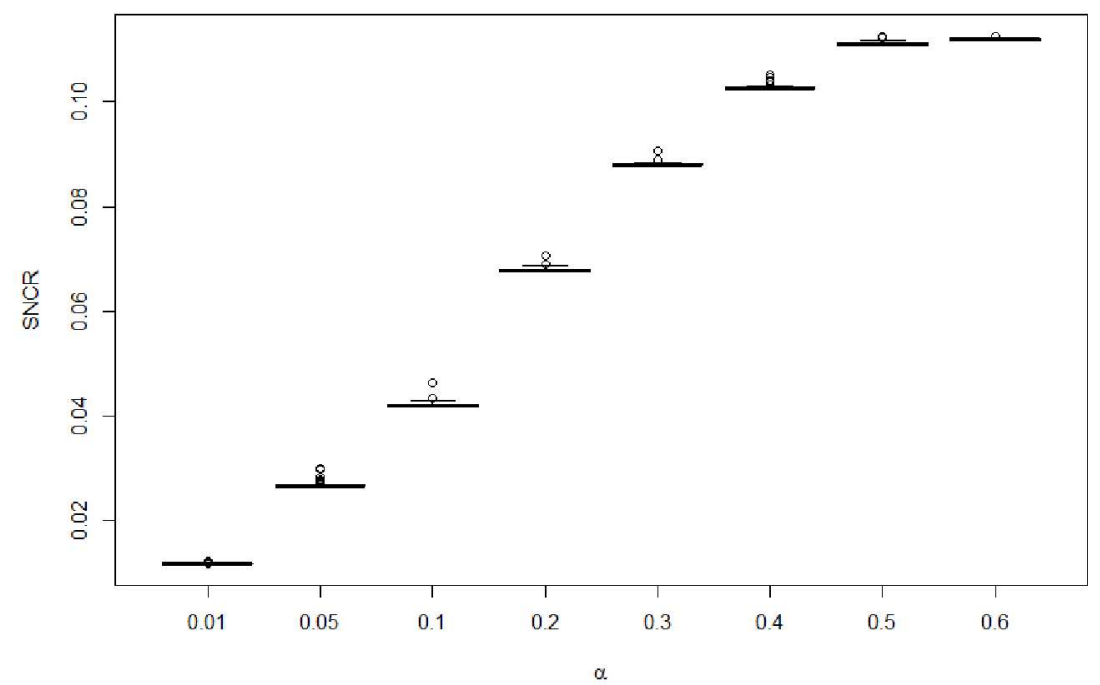

Figure 3: Theoretically optimal SNCR versus $\alpha(\mathrm{N}=180)$

From Figure 3, it is clear that the $\mathrm{SNCR}_{\text {opt }}$ depends largely on $\alpha$, while $\kappa$ and $\eta$ do not have much impact on it. Also it is worthwhile noting that the 


\begin{tabular}{|l|l|l|l|l|l|l|l|l|}
\hline $\boldsymbol{\alpha}$ & 0.01 & 0.050 & 0.1 & 0.2 & 0.3 & 0.4 & 0.5 & 0.6 \\
\hline min & 0.0118 & 0.0266 & 0.0417 & 0.0674 & 0.0878 & 0.1025 & 0.1108 & 0.1118 \\
\hline mean & 0.119 & 0.0270 & 0.0422 & 0.0679 & 0.0883 & 0.1029 & 0.1111 & 0.1120 \\
\hline median & 0.0118 & 0.0267 & 0.0419 & 0.0677 & 0.0880 & 0.1026 & 0.1109 & 0.1120 \\
\hline max & 0.0123 & 0.0300 & 0.0462 & 0.0707 & 0.0907 & 0.1051 & 0.1125 & 0.1124 \\
\hline
\end{tabular}

Table 3: Summary of theoretical optimal SNCR versus. $\alpha(\mathrm{N}=180)$

total sample size $N$ plays an important role in the derivation of $\sigma_{\hat{\pi}(d)}$, as $N$ increases, the variance becomes smaller. Hence we can say that the $\mathrm{SNCR}_{\text {opt }}$ can be estimated well if we have knowledge about $\alpha$ and $N$.

Thus while we have not investigated the mathematical reasoning behind it, we conjecture that $\alpha$ plays a crucial role in the determination of the optimal SNCR as $\hat{\alpha}$ itself has variability and $V(\hat{\alpha})$ is much smaller when $\alpha$ is small.

\subsection{Relationship between $\frac{d_{2}}{d_{3}}$ and $\eta$ under optimal de- signs}

The strong relationship between $\mathrm{SNCR}_{\text {opt }}$ and $\alpha$ is not the only empirical result found in this study. The results also suggest that there is a strong correlation between the ratio of the optimal intermediate and maximum dose, and the Hill coefficient $\eta$.

The plot of $s=\frac{d_{2, o p t}}{d_{3, \text { opt }}}$ against $\eta$ is given in the left of Figure 4. A Box-Cox transformation is used to linearize the relationship, and the transformed plot is given in the right of Figure 4. The least squares fit for $s$ against $\eta$ is given by:

$$
s^{2.315}=-0.098+0.051 \eta \text { or } s=(-0.098+0.051 \eta)^{1 / 2.315}
$$


with corresponding coefficient of determination of 0.9783 .

Figure 5 gives the results of the model's diagnostic check. The plot of residuals vs. fitted values does not show a strong trend. At the same time, the middle part of the normal QQ plot is well-behaved. Hence there is no strong reason to suspect the model's inadequacy.
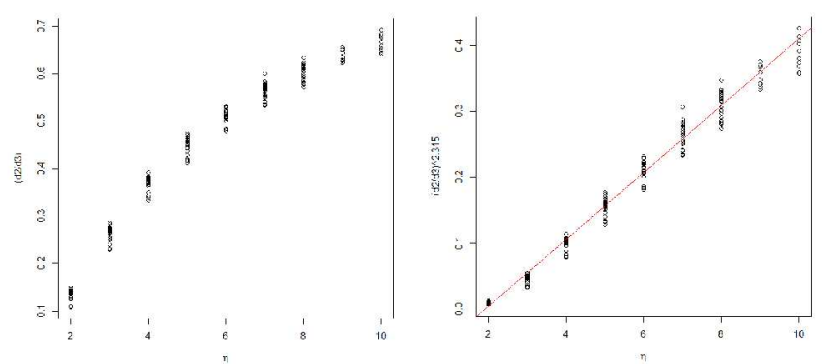

Figure 4: Optimal ratio $s$ versus $\eta$
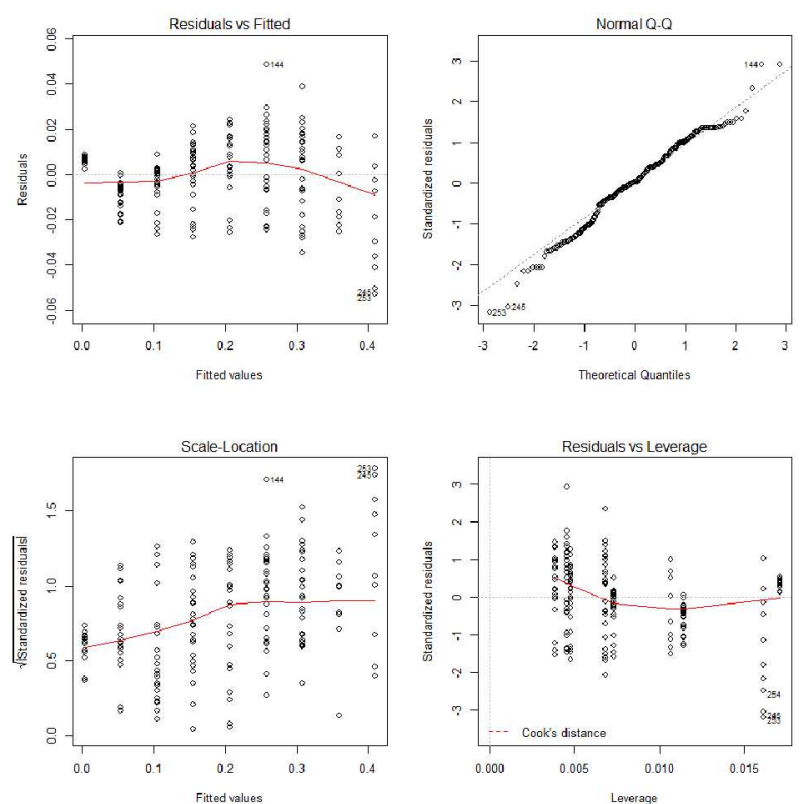

Figure 5: Diagnostic check for the model given by equation (13) 


\subsection{Sub-optimal designs and their performance}

\subsubsection{How to evaluate the performance of a sub-optimal design for SNCR?}

Suppose that we have a parameter $\theta$ that we wish to estimate. In general, the relative efficiency of estimator $\hat{\theta}_{1}$ to $\hat{\theta}_{2}$ is given by

$$
\text { efficiency }=\frac{V\left(\hat{\theta}_{1}\right)}{V\left(\hat{\theta}_{2}\right)}
$$

By definition, under a fixed sample size $N$, a point estimate of SNCR under the optimal design $\left(\mathrm{SNCR}_{\text {opt }}\right)$ would be less than an estimate given by a sub-optimal design $\left(\mathrm{SNCR}_{\text {sub }}\right)$. In other words, we cannot simply compare the variance of two SNCR estimators to evaluate the efficiency of a suboptimal design.

However, even under a sub-optimal design, a sufficiently increased sample size, say $N_{\text {sub }}$, would enable us to obtain $\mathrm{SNCR}_{\text {opt }}$ under the sample size $N$. In other words, it is possible to obtain an equality

$$
\operatorname{SNCR}\left(\boldsymbol{\theta}, \boldsymbol{d}_{\text {opt }}, \boldsymbol{c}_{\text {opt }}, N\right)=S N C R\left(\boldsymbol{\theta}, \boldsymbol{d}_{\text {sub }}, \boldsymbol{c}_{\text {sub }}, N_{\text {sub }}\right)
$$

Hence, we define the efficiency of the sub-optimal design by:

$$
\text { efficiency }=m=\frac{N}{N_{\text {sub }}}
$$

such that (14) holds. Note that the reciprocal of the efficiency $m^{-1}$ yields how large does the sample size needs to be for a sub-optimal design to attain the SNCR based on the optimal design. In what follows we describe the 
approach for determining the efficiency $m$ and an alternative sample size $N_{\text {sub }}$.

In section 2.1.3, we derived the variance at a given excess risk $\hat{\pi}_{0}$, which is given by

$$
V\left(\hat{\pi}_{0}\right)=\boldsymbol{\Pi}^{T} \boldsymbol{X}_{(\hat{\boldsymbol{\theta}})}^{-1} \boldsymbol{\Pi}
$$

where only $\boldsymbol{X}_{(\hat{\boldsymbol{\theta}})}^{-1}$ is dependent on the sample size $N$. In particular, we can rewrite $\boldsymbol{X}_{(\hat{\boldsymbol{\theta}})}^{-1}$ as

$$
\boldsymbol{X}_{(\hat{\boldsymbol{\theta}})}^{-1}=\frac{1}{N} \dot{\boldsymbol{X}}_{(\hat{\boldsymbol{\theta}})}^{-1}
$$

where

then (10) becomes

$$
\dot{\boldsymbol{X}}_{(\hat{\boldsymbol{\theta}})}=\sum_{i=1}^{t} \frac{c_{i} P_{r}\left(d_{i}\right) P_{s}\left(d_{i}\right)}{P\left(d_{i}\right)\left[1-P\left(d_{i}\right)\right]}
$$

$$
\begin{aligned}
V\left(\hat{\pi}_{0}\right) & =\frac{1}{N} \boldsymbol{\Pi}^{T} \dot{\boldsymbol{X}}_{(\hat{\boldsymbol{\theta}})}^{-1} \boldsymbol{\Pi} \Rightarrow \\
\sigma_{\hat{\pi}_{0}} & =\frac{1}{\sqrt{N}}\left(\boldsymbol{\Pi}^{T} \dot{\boldsymbol{X}}_{(\hat{\boldsymbol{\theta}})}^{-1} \boldsymbol{\Pi}\right)^{1 / 2}
\end{aligned}
$$

Now, let us denote $\sigma_{\hat{\pi}_{0}}$ from the optimal design by $\sigma_{\hat{\pi}_{0}, \text { opt }}$. Similarly, let $\sigma_{\hat{\pi}_{0}, s u b}$ represent the standard error given from a sub-optimal design.

Then we can solve for $N_{\text {sub }}$ and $m$ as

$$
\begin{aligned}
\operatorname{SNCR}\left(\boldsymbol{\theta}, \boldsymbol{d}_{\text {opt }}, \boldsymbol{c}_{\text {opt }}, N\right) & =\operatorname{SNCR}\left(\boldsymbol{\theta}, \boldsymbol{d}_{\text {sub }}, \boldsymbol{c}_{\text {sub }}, N_{\text {sub }}\right) \Leftrightarrow \\
\lambda\left(\sigma_{\hat{\pi}_{0}, \text { opt }} \mid N\right) & =\lambda\left(\sigma_{\hat{\pi}_{0}, \text { sub }} \mid N_{\text {sub }}\right) \Leftrightarrow \\
\frac{1}{\sqrt{N}}\left(\boldsymbol{\Pi}^{T} \dot{\boldsymbol{X}}_{(\boldsymbol{\theta}), \text { opt }}^{-1} \boldsymbol{\Pi}\right)^{1 / 2} & =\frac{1}{\sqrt{N}_{\text {sub }}}\left(\boldsymbol{\Pi}^{T} \dot{\boldsymbol{X}}_{(\boldsymbol{\theta}), \text { sub }}^{-1} \boldsymbol{\Pi}\right)^{1 / 2} \Leftrightarrow \\
\frac{N}{N_{\text {sub }}} & =m=\frac{\boldsymbol{\Pi}^{T} \dot{\boldsymbol{X}}_{(\boldsymbol{\theta}), \text { opt }}^{-1} \boldsymbol{\Pi}}{\boldsymbol{\Pi}^{T} \dot{\boldsymbol{X}}_{(\boldsymbol{\theta}), \text { sub }}^{-1} \boldsymbol{\Pi}}
\end{aligned}
$$


In other words, the efficiency $m$ can be determined by dividing the standard error at $S N C R_{\text {opt }}$ under the optimal design by the standard error at $S N C R_{\text {opt }}$ under the sub-optimal design with a total sample size of $N$.

In addition to efficiency, we shall also consider the ratio $r=\frac{S N C R_{\text {opt }}}{S N C R_{s u b}}$ as a secondary measure of the performance of sub-optimal designs.

\subsection{Four sub-optimal designs considered in this study}

In this section, note that we only consider theoretically optimal SNCR estimates.

In order to optimize the experimental design for SNCR, we need to have some knowledge of the Hill parameters $\boldsymbol{\theta}$ prior to running the experiment. Since this information is not readily available in practice, we must rely on sub-optimal designs with fixed dose levels and proportions of allocation. In particular, we investigate sub-optimal designs where the lowest experimental dose level is set to be the control, and the maximum dose level used in the optimal design is set to be the maximum sub-optimal dose level.

There are other advantages in employing sub-optimal designs instead of the optimal design. First, if a sub-optimal design has four or more dose levels, we can apply a goodness-of-fit test of the derived Hill model against a saturated model. Also, sub-optimal designs with a greater number of dose levels provide a better chance of capturing the lower and upper inflection points of the dose-response relationship, as well as the NOAEL if the MTD is very small. 
In this study, we investigate the effect of the design parameters on the estimation of the SNCR under different combinations of Hill model parameters. In particular, four sub-optimal designs ginen in Table 4 are employed for comparison purposes.

\begin{tabular}{|l|l|l|}
\hline Design & Number of dose levels & Experimental dose levels \\
\hline $\mathrm{SD}_{1}$ & 3 & $\left(0, \frac{1}{2}, 1\right)$ \\
\hline $\mathrm{SD}_{2}$ & 4 & $\left(0, \frac{1}{3}, \frac{2}{3}, 1\right)$ \\
\hline $\mathrm{SD}_{3}$ & 4 & $\left(0, \frac{1}{4}, \frac{1}{2}, 1\right)$ \\
\hline $\mathrm{SD}_{4}$ & 6 & $\left(0, \frac{1}{6}, \frac{1}{3}, \frac{1}{2}, \frac{2}{3}, \frac{5}{6}, 1\right)$ \\
\hline
\end{tabular}

Table 4: Summary of the four sub-optimal designs used in the study

Note that all designs have equal proportions of allocation at each dose level, and an experimental dose level of one corresponds to the MDT under the optimal design.

\subsubsection{Results and their implications on the performances of sub- optimal designs}

Median efficiencies of sub-optimal designs In our study, there is no sub-optimal design that consistently performed best amongst the four designs described above. Figure 6 shows the median efficiencies with a fixed background response rate of 0.1 . The reciprocals of these efficiencies are given in Table 5 .

From Figure 6, we observe that the efficiency of each design changes based on $\eta$. For instance, $\mathrm{SD}_{1}$ outperforms the other three designs for $\eta$ between 5 to 7 ; however, its efficiency is relatively poor for small or large values of $\eta$. 


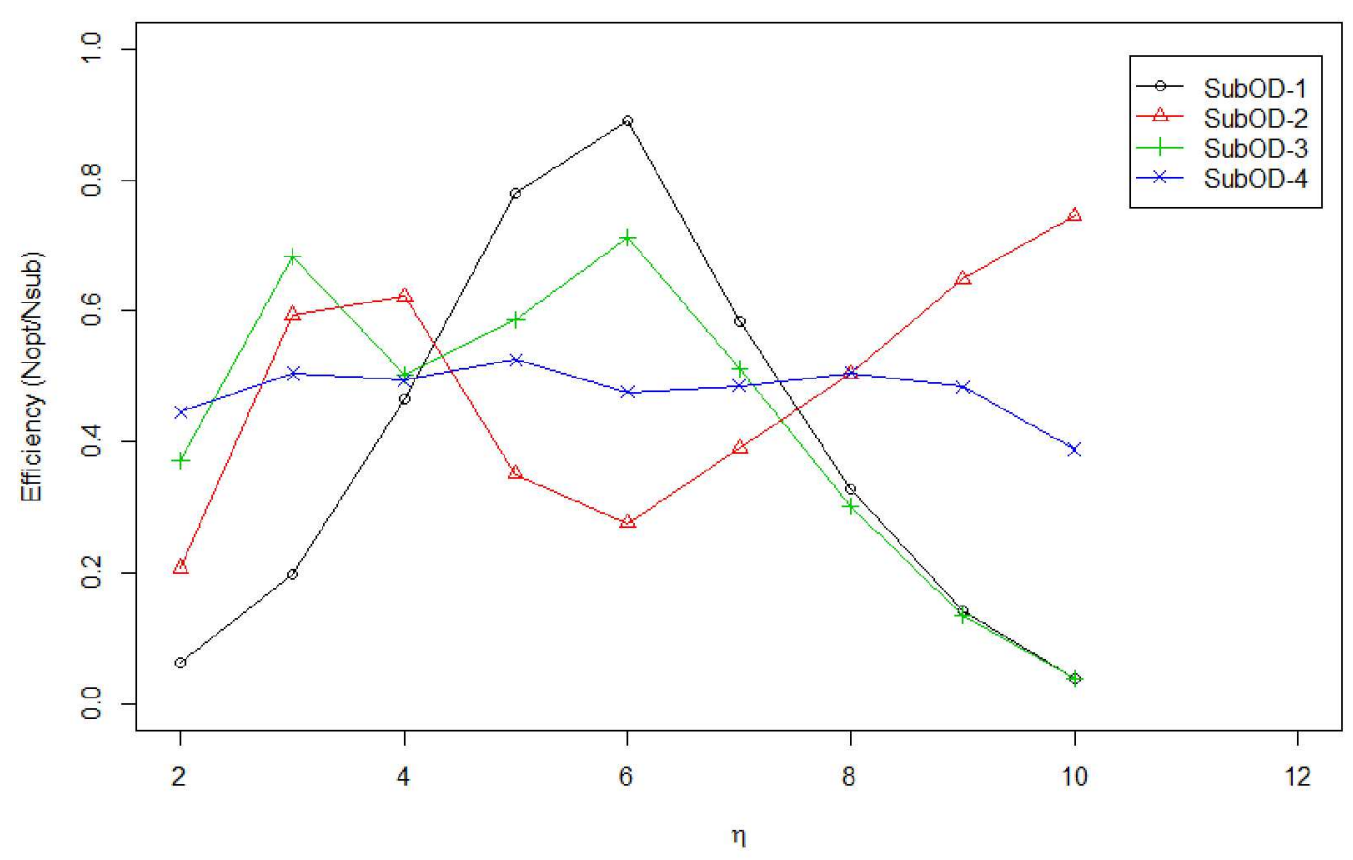

Figure 6: Median efficiencies of sub-optimal designs $(\alpha=0.10)$

\begin{tabular}{|l|l|l|l|l|l|l|l|l|l|}
\hline Design $\backslash \eta$ & 1 & 2 & 3 & 4 & 5 & 6 & 7 & 8 & 9 \\
\hline $\mathrm{SD}_{1}$ & 15.78 & 5.05 & 2.15 & 1.28 & 1.12 & 1.71 & 3.05 & 7.02 & 25.21 \\
\hline $\mathrm{SD}_{2}$ & 4.80 & 1.68 & 1.61 & 2.85 & 3.62 & 2.56 & 1.98 & 1.54 & 1.34 \\
\hline $\mathrm{SD}_{3}$ & 2.69 & 1.46 & 1.99 & 1.71 & 1.40 & 1.95 & 3.31 & 7.35 & 25.74 \\
\hline $\mathrm{SD}_{4}$ & 2.24 & 1.98 & 2.02 & 1.90 & 2.10 & 2.06 & 1.98 & 2.62 & 2.57 \\
\hline
\end{tabular}

Table 5: Reciprocals of median efficiencies $(\alpha=0.10)$

Recall that equation (13) from Section 3.4 suggests that there is a strong correlation between the ratio $s=\frac{d_{2}}{d_{3}}$ and the Hill coefficient $\eta$. The optimal ratio $s$ against different values of $\eta$ is presented in Table 6 .

\begin{tabular}{|l|l|l|l|l|l|l|l|l|l|}
\hline $\boldsymbol{\eta}$ & 2 & 3 & 4 & 5 & 6 & 7 & 8 & 9 & 10 \\
\hline $\boldsymbol{s}$ & 0.058 & 0.284 & 0.378 & 0.448 & 0.506 & 0.557 & 0.602 & 0.643 & 0.680 \\
\hline
\end{tabular}

Table 6: Optimal ratio $s$ at different values of $\eta$

From Table 6, we observe that the optimal ratio $s$ increases monotonically 
as $\eta$ increases. As given in Table 4 , the ratio $s$ of $\mathrm{SD}_{1}$ is fixed at 0.5 , which implies that the maximum efficiency is obtained when $\eta$ is about 6 , and its efficiency decreases as $\eta$ moves farther from 6 .

The second sub-optimal design performs well when $\eta$ is either 4, 9, or 10 . For $\eta=4$ the $d_{2}$ of 0.33 captures the dose levels of the optimal design well. When $\eta$ is either 9 or 10 , the higher intermediate dose of 0.67 well represents the optimal ratio $s$ of 0.643 and 0.680 . The loss in efficiency is also caused by the fixed proportions of allocation, as well as the reduced sample size per group due to the increase in the number of the experimental dose levels (for $\mathrm{SD}_{2}, \mathrm{SD}_{3}$, and $\left.\mathrm{SD}_{4}\right)$. Note that the efficiency for $\mathrm{SD}_{4}$ is the most consistent. This is due to the fact that each of the experimented dose levels represents the optimal ratio $s$ for different values of $\eta$. However, the reduced sample size in each dose level results in lower efficiency compared to other sub-optimal designs under consideration.

It is also worthwhile noting that while the general trend of the efficiency holds at different values of $\alpha$, there is a considerable change in relative performance at higher $\alpha$. In particular, $\mathrm{SD}_{2}$ begins to outperform the other designs at slightly lower $\eta$. This tendency is demonstrated in Figure 7 below with $\alpha$ at 0.50 .

Median Ratios of $\mathbf{S N C R}_{o p t}$ versus $\mathbf{S N C R}_{s u b}$ In general, the plot of median ratios of $r=\frac{S N C R_{\text {opt }}}{S N C R_{\text {sub }}}$ versus $\eta$ is almost identical to the median efficiencies given in the previous section. Figure 8 gives the relationship between $r$ and $\eta$ under $\alpha=0.01$. It should also be noted that while the 


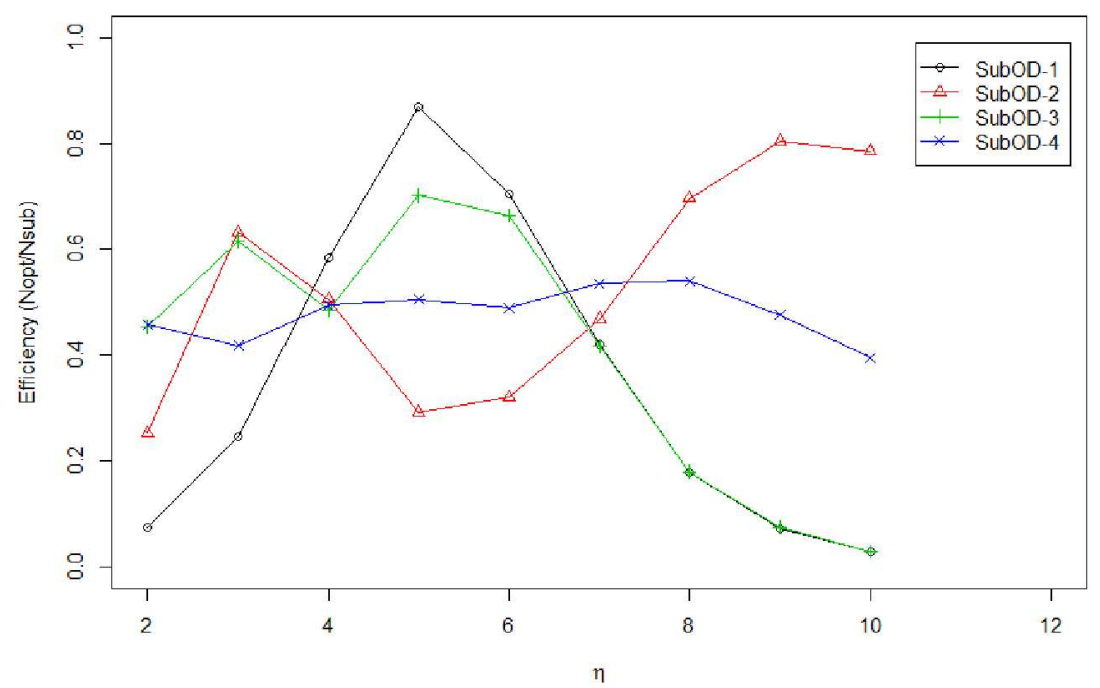

Figure 7: Median efficiencies of sub-optimal designs $(\alpha=0.50)$

shape of the relationships remain the same as $\alpha$ increases, the median ratios also increase. This can be seen by comparing Figures 8 and 9 .

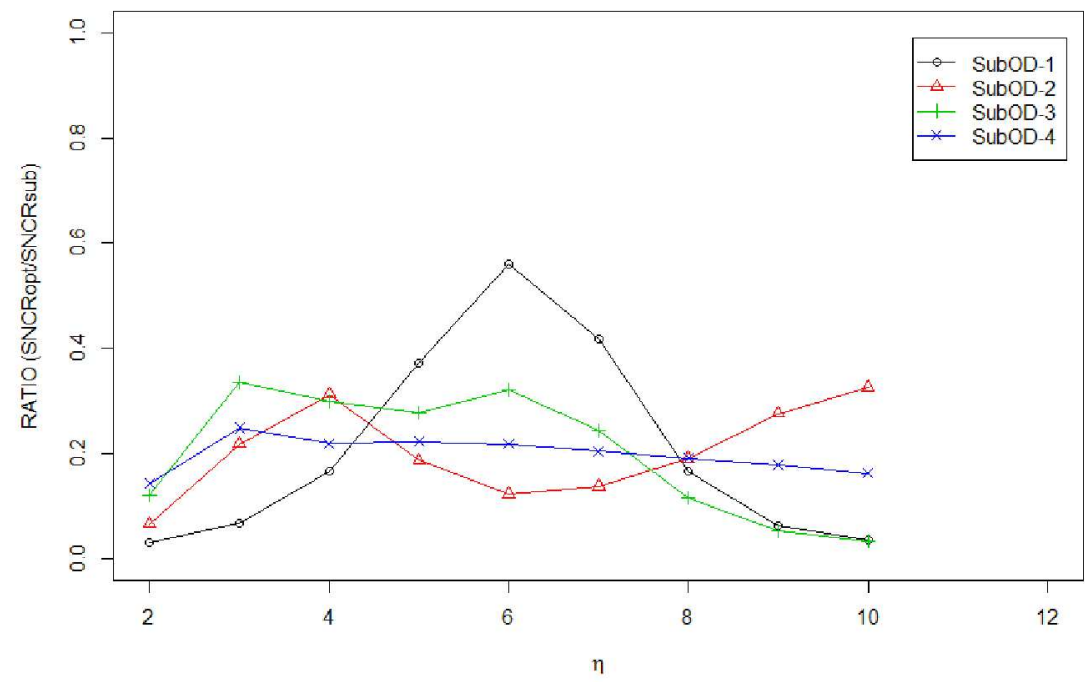

Figure 8: Median ratios of sub-optimal designs $(\alpha=0.01)$

Given the performance of the SNCR based on the sub-optimal designs, it is clear that, regrettably, we cannot employ $S N C R_{\text {sub }}$ as a PoD for health 


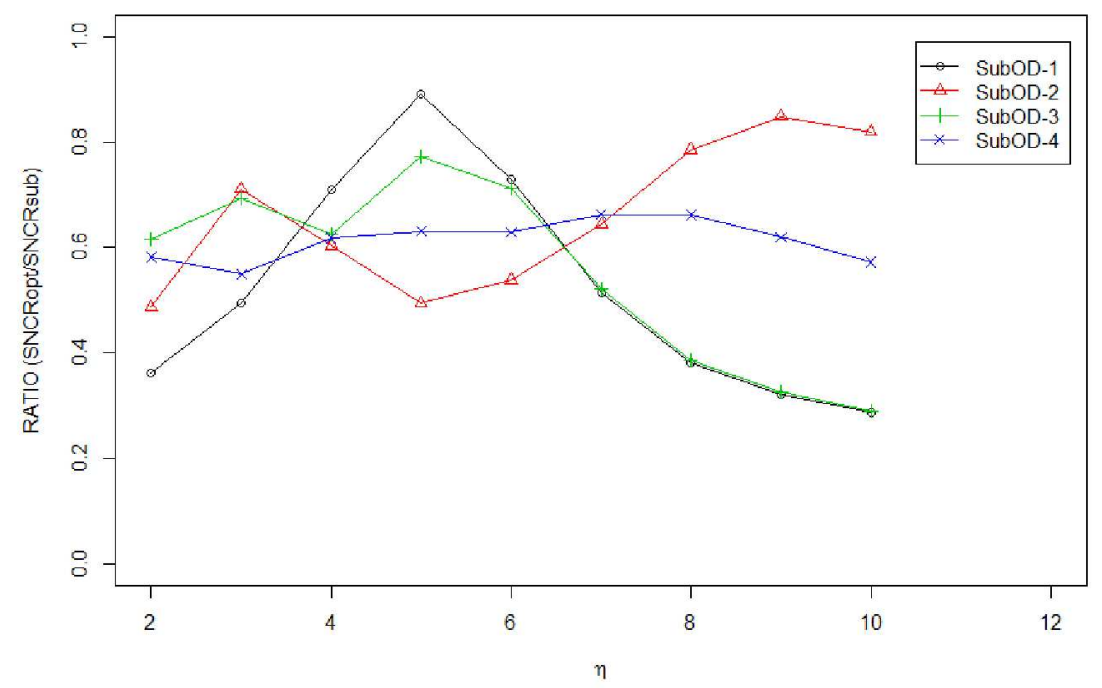

Figure 9: Median ratios of sub-optimal designs $(\alpha=0.50)$

risk assessment as it would provide a false sense of safety. However, we also know that the sample size $N$ and $\alpha$ have important roles in the determination of SNCR. Thus we now investigate how the change in the total sample size affects the estimation of SNCR.

\subsubsection{The effect of the sample size on SNCR $_{\text {opt }}$}

As the total sample size $N$ increases, the asymptotic variance of an estimated PoD and corresponding excess risk decrease. It is only natural then that by running two identical experiments with sample sizes $N_{1}<N_{2}$, the SNCR estimate based on $N_{2}$ would be smaller than the one based on $N_{1}$. Figure 10 and Table ?? shows the change in median $S N C R_{\text {opt }}$ based on different sample sizes ranging from $N=90$ to 540 .

At each level of $\alpha$, it is clear that the larger the sample size, the smaller the resulting estimated SNCR. Furthermore, we observe that within the investigated $\alpha$ values, the increase in $\alpha$ also results in an increase in SNCR. 
However, the interaction effect of $N$ and $\alpha$ on SNCR is not so clear. For $N \leq 270$ the change in SNCR with respect to $\alpha$ is concave, while $N=360$ gives an almost linear relationship. When $N \geq 450$ the relationship is characterized by convex curve.

Given the extent that SNCR is affected due to the change in $N$, another question that arises relates to what is an appropriate sample size to be used for deriving this quantity. (Note that $\lim _{N \rightarrow \infty} S N C R=0$ )

On a side note, it is also worth pointing out that the increase in the sample size does not affect the optimal dose levels, but it changes the proportions of allocations at each dose level. 


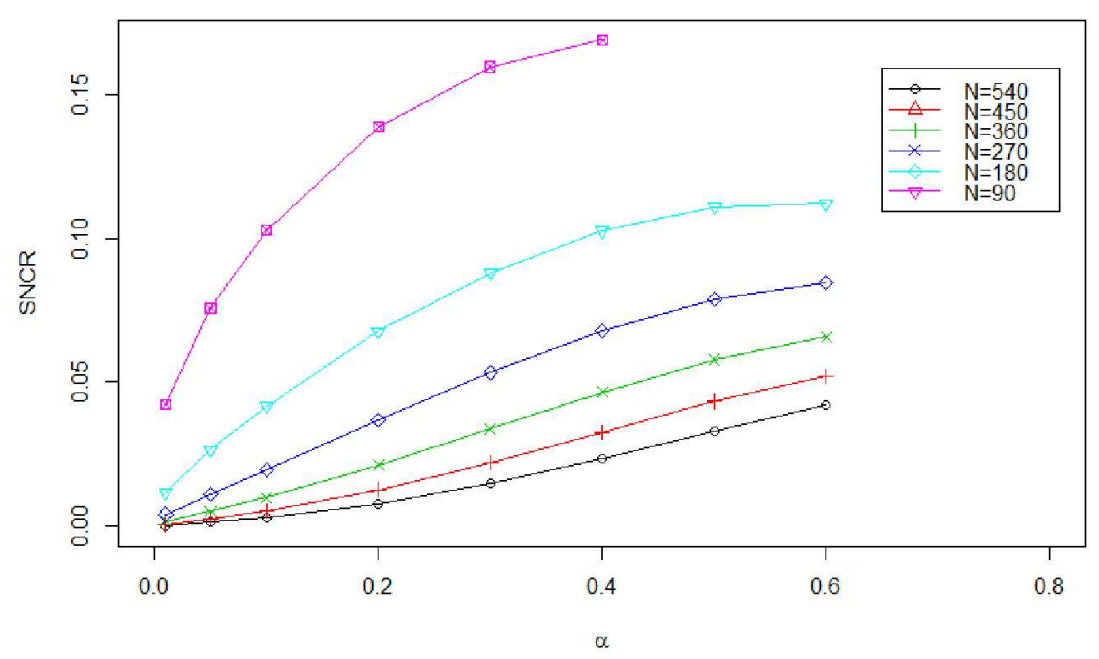

Figure 10: Median optimal SNCR versus $N$

\begin{tabular}{|l|l|l|l|l|l|l|l|l|}
\hline$N, \alpha$ & $\mathbf{0 . 0 1}$ & $\mathbf{0 . 0 5}$ & $\mathbf{0 . 1}$ & $\mathbf{0 . 2}$ & $\mathbf{0 . 3}$ & $\mathbf{0 . 4}$ & $\mathbf{0 . 5}$ & $\mathbf{0 . 6}$ \\
\hline $\mathbf{9 0}$ & $4.2^{-2}$ & $7.6^{-2}$ & $1.0^{-1}$ & $1.4^{-1}$ & $1.6^{-1}$ & $1.7^{-1}$ & $N A$ & $N A$ \\
\hline $\mathbf{1 8 0}$ & $1.2^{-2}$ & $2.7^{-2}$ & $4.2^{-2}$ & $6.8^{-2}$ & $8.8^{-2}$ & $1.0^{-1}$ & $1.1^{-1}$ & $1.1^{-1}$ \\
\hline $\mathbf{2 7 0}$ & $4.2^{-2}$ & $1.1^{-2}$ & $2.0^{-2}$ & $3.7^{-2}$ & $5.3^{-2}$ & $6.8^{-2}$ & $7.8^{-2}$ & $8.5^{-2}$ \\
\hline $\mathbf{3 6 0}$ & $1.7^{-3}$ & $5.2^{-3}$ & $1.0^{-2}$ & $2.1^{-2}$ & $3.4^{-2}$ & $4.7^{-2}$ & $5.8^{-2}$ & $6.6^{-2}$ \\
\hline $\mathbf{4 5 0}$ & $7.9^{-4}$ & $2.6^{-3}$ & $5.4^{-3}$ & $1.3^{-2}$ & $2.2^{-2}$ & $3.3^{-2}$ & $4.3^{-2}$ & $5.2^{-2}$ \\
\hline $\mathbf{5 4 0}$ & $3.9^{-4}$ & $1.4^{-3}$ & $3.1^{-3}$ & $8.0^{-3}$ & $1.5^{-2}$ & $2.3^{-2}$ & $3.3^{-2}$ & $4.2^{-2}$ \\
\hline
\end{tabular}

Table 7: Theoretical optimal SNCR versus total sample size $N$ 


\section{Optimal designs and the NTP database}

In 1978, the Secretary of the Department of Health and Human Services launched the National Toxicology Program (NTP) in order to determine the RfDs for potentially hazardous chemicals that are found in industrial and consumer products. In the present study, we shall explore the feasibility of the optimal designs for SNCR using 788 carcinogenesis and toxicity studies employing rats. These experiments are part of the data that were abstracted on 13-14 January 2009 by Sand and others. To summarize our findings:

(a) $426(54 \%)$ of the experiments had virtually zero background risk.

(b) More than $90 \%$ of the experiments had an estimated $\kappa>M D T$.

(c) 699 experiments (88.7\%) had an estimated $\eta$ of one.

In cases of (a) and (c), we cannot obtain three-dose optimal designs for SNCR as the estimated Hill model can be described by at most two parameters. Also, as discussed earlier, when the estimated $\kappa$ lies in outside of the experimental range, the derivation of the practical optimal design is nearly impossible. These considerations imply that the implementation of the optimal design for SNCR in practice is not feasible. In fact, there are only 19 experiments that are classified in none the above three criteria.

\subsection{An Illustrative Example from the NTP database}

In this particular experiment, the agent under investigation was C.I. Acid Red 114: a dye used for colouring wool and leather materials. The study 
consisted of 200 male rats, where the response of interest was the presence/absence of malignant tumours on any organ. The SNCD and SNCR based on the actual experiment are summarized in Table 8. Furthermore, the restricted optimal design and the resulting SNCD and SNCR are given in Table 9.

Figure 11 shows the estimated dose-response curve. Observed response rates at dose levels used in the experiment are given in blue circles, while grey vertical lines represent the restricted optimal dose levels.

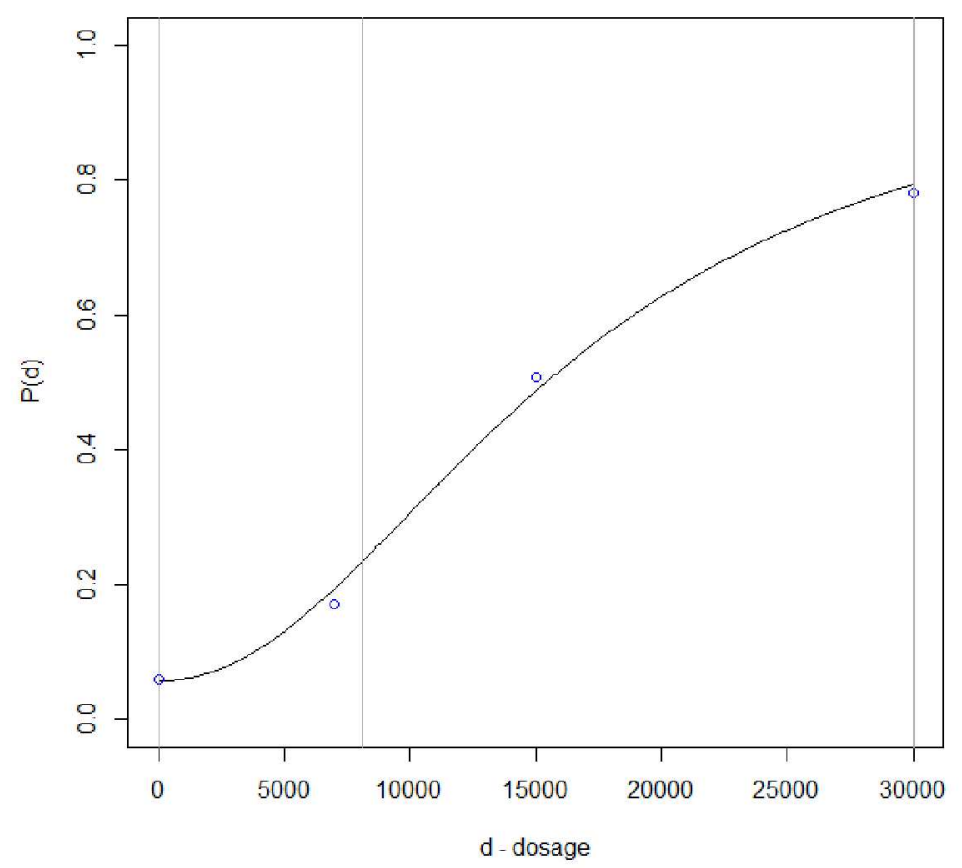

Figure 11: Dose-response curve for C.I. Acid Red 114

As shown in Figure 11, the actual experiment captures a sigmoidal doseresponse curve, which is found to be one of the key elements in deriving the theoretical optimal design. From the actual experiment, the SNCR is found 
to be 0.0655 . Assuming that the estimated Hill model parameters are the true parameters, the optimal design would yield an SNCR of 0.0327.

This example illustrates some of the difficulties that the SNCR faces. Firstly, the SNCR based on the actual experiment is twice as large as what could have been derived under the optimal design. Secondly, even though we were able to capture a sigmoidal dose-response curve in the experimental range, we could not obtain the theoretical optimal design due to a small MDT.

\begin{tabular}{|l|llll|}
\hline Actual Experiment & 200 & & & \\
\hline Total Sample Size $(N)$ & 0.057 & 16334 & 2.10 & \\
\hline MLE of Hill parameters $(\hat{\boldsymbol{\theta}}=(\alpha, \kappa, \eta))$ & 0 & 7000 & 15000 & 30000 \\
\hline Experimented Dose Levels $(\boldsymbol{d})$ & 50 & 35 & 65 & 50 \\
\hline Sample Sizes $(\boldsymbol{n})$ & 3 & 6 & 33 & 39 \\
\hline Number of Observed Responses $(\boldsymbol{y})$ & 0.06 & 0.17 & 0.51 & 0.78 \\
\hline Observed Response rates $(\boldsymbol{p})$ & 4720 & & & \\
\hline SNCD & 0.0655 & & & \\
\hline SNCR & & & & \\
\hline
\end{tabular}

Table 8: Summary of results from observed experiment involving C.I. Acid Red 114

\begin{tabular}{|l|lll|}
\hline \multicolumn{4}{|l|}{ Restricted Optimal Experiment } \\
\hline Total Sample Size $(N)$ & 200 & & \\
\hline MLE of Hill parameters $(\hat{\boldsymbol{\theta}}=(\alpha, \kappa, \eta))$ & 0.057 & 16334 & 2.10 \\
\hline Experimented Dose Levels $(\boldsymbol{d})$ & 0 & 8100 & 30000 \\
\hline Sample Sizes $(\boldsymbol{n})$ & 51 & 111 & 38 \\
\hline SNCD & 3330 & & \\
\hline SNCR & 0.0327 & & \\
\hline
\end{tabular}

Table 9: Summary of restricted optimal design associated with the results in Table 8 


\section{Conclusions and Discussions}

The concept of SNCD was developed in the hopes of overcoming the difficulty in choosing an appropriate increase in risk to be used for the determination of the endpoints to be used in health risk assessment. In 2011, Sand et al. introduced the concept of the SNCD to represent a dose level such that the increase in risk is relatively large compared to the variability around it so that the added risk can be reliably detected. The U.S. EPA and the EFSA recommend that the method of $\mathrm{BMD}$ be used for determining the PoD for human exposure guidelines; however, due to practical constraints, the choice of BMR used in practice does not have a strong underlying statistical theory to claim that the BMR represents an acceptable/tolerable amount of increase in risk. The suggested choices of $1 \%, 5 \%$, and $10 \%$ as BMRs (depending on the sensitivity of the experiment), from a statistical theory perspective, are rather arbitrary (nevertheless these choices are easy to conceptualize). In their paper, Sand et al. (2011) defined the SNCD to be a dose such that the excess risk at SNCD is equivalent to the width (or $2 / 3$ of it) of the $90 \%$ confidence interval about the absolute risk at SNCD; however, this choice of SNR also seems arbitrary.

A new SNR is introduced in this study in the hopes of determining the minimum PoD that would yield a statistically significant increase in risk. For this reason, we set the SNCD to be a dose level such that, in a given experiment, the excess risk at SNCD can be considered significant with $90 \%$ confidence. 
The main objective of this thesis is to develop the optimal design for the SNCR and study its behaviour under a variety of dose-response relationships. The optimality criterion of the design is given so that it minimizes the variance of SNCR estimate under known Hill model parameters $\boldsymbol{\theta}$. This also implies that the SNCR under the optimal design is locally minimized.

In section 2, we developed the theoretical optimal design for SNCR and described how to find it numerically. In section 3, a study is conducted using a number of combinations of Hill model parameters to obtain theoretical and restricted optimal designs. The SNCR given under the theoretical optimal designs are compared with four sub-optimal designs. Of the four types of observable dose-response relationships considered here (i.e., sigmoidal, concave, near linear, and convex), it was found that the derivation of theoretical optimal designs was possible only under the experiments with a sigmoidal dose-response relationships with MDT corresponding to an absolute risk of 98\%. An interesting finding is that the SNCR, under theoretical optimal design, is virtually independent of $\kappa$ and $\eta$, and only depends on $\alpha$ and the total sample size $N$. As $N$ increases, the resulting SNCR decreases. At the same time, the increase in $\alpha$ results in an increase in SNCR as well, for $\alpha$ as large as $60 \%$. Another finding is that the intermediate optimal dose level would always be less than $\kappa$.

From the NTP datasets abstracted by Sand and others, it is found that the application of theoretical or restricted optimal designs for the Hill model are difficult in practice. One reason is that the MTD often corresponds to a very small increase in risk; hence only the lower tail of the dose-response 
relationship would be observed in this instance. Also, when $\alpha$ is very small, the optimal proportion of allocation for the control group is virtually zero, leading it to be a two-dose optimal design. Similarly, when $\kappa$ is one, the Hill model becomes a two-parameter model, indicating that its optimal design only requires two dose levels. One of the advantages of the Hill model is that its parameters are easy to interpret; however, given the observed requirements for the optimal design to capture $\kappa$ in between the intermediate dose level and the MDT, the practical constraint on MDT prohibits the construction of the theoretical optimal design in many carcinogenic experiments.

A number of obstacles must be overcome prior to applying the SNCR in practice. Some of these may be less problematic in other dose-response relationship models such as Weibull and one-hit, as these models do not require estimation of $\kappa$ as a model parameter. Another difficulty is that under the new definition of SNR, a relatively smaller sample size, or a design far from optimal may lead to a larger SNCR, which may provide a false sense of safety.

Many of the findings from this study would apply to the original definition of SNCD as well. Since the $90 \%$ confidence interval would also be wider for experimental designs that are far from optimal; it would also provide a false sense of safety.

Even though the RfD based on $\mathrm{SNCD}_{\text {orig }}$ using linear extrapolation would increase as the sample size increases (Sand et al., 2011), the choice of SNR is crucial as the smaller the SNR, the greater would be the resulting RfD when $\eta>1$. 
The concept of SNCD to incorporate the concept of signal-to-noise ratio to determine an appropriate BMR to be used for setting human exposure guidelines has strong intuitive appeal. However, as summarized above, the method of SNCD faces a number of difficulties, and further investigation into the underlying statistical theory is required in order to overcome some of the obstacles discovered here. 


\section{References}

[1] U.S. EPA (U.S. Environmental Protection Agency). Benchmark dose (bmd) methodology. http://www.epa.gov/ncea/bmds/bmds_training/ methodology/intro.htm/, 2014.

[2] U.S. EPA (U.S. Environmental Protection Agency). Epa risk assessment glossary. http://www.epa.gov/risk_assessment/glossary . $\mathrm{htm} /, 2014$.

[3] EFSA (European Food Safety Authority). Guidance of the scientific committee on a request from efsa on the use of the benchmark dose approach in risk assessment. EFSA Journal, 1150:1-72, 2009.

[4] H. Chernoff. Locally optimal designs for estimating parameters. Topics in Applied Statistics, pages 586-602, 1952.

[5] D. Krewski, J. Kover, and M. Bickis. Optimal experimental designs for low dose extrapolation. Topics in Applied Statistics, pages 167-191, 1984.

[6] D. Krewski and J. V. Ryzin. Dose response models for quantal response toxicity data. Statistics and Related Topics, pages 201-231, 1981.

[7] K. S. Krump. A new method for determining allowable daily intakes. Foundamental and Applied Toxicology, 4:854-871, 1984.

[8] A. J. Lehman and O. G. Fitzhugh. 100-fold margin of safety. 18:33-35, 1954 
[9] S. Sand, C. J. Portier, and D. Krewski. A signal-to-noise crossover dose as the point of departure for health risk assessment. Environmental Health Perspectives, 119:1766-1774, 2011. 


\section{A Proofs and verifications}

\section{A.1 The Newton-Raphson method for estimating the Hill model parameters}

Suppose that the experiment is set up as described in section 2.1.1, which gives

$l(\boldsymbol{\theta})=\sum_{i=1}^{t}\left\{x_{i} \ln \left[\alpha+(1-\alpha)\left(\frac{d_{i}^{\eta}}{d_{i}^{\eta}+\kappa^{\eta}}\right)\right]+\left(n_{i}-x_{i}\right) \ln \left[(1-\alpha)\left(1-\frac{d_{i}^{\eta}}{d_{i}^{\eta}+\kappa^{\eta}}\right)\right]\right\}$

For simplicity, let

$$
\begin{gathered}
B_{i}=\left(\frac{d_{i}^{\eta}}{d_{i}^{\eta}+\kappa^{\eta}}\right) \\
C_{i}=\frac{\partial A_{i}}{\partial \kappa}=\frac{\partial}{\partial \kappa}\left(\frac{d_{i}^{\eta}}{d_{i}^{\eta}+\kappa^{\eta}}\right)=\frac{-\eta d_{i}^{\eta} \kappa^{\eta-1}}{\left(d_{i}^{\eta}+\kappa^{\eta}\right)^{2}}
\end{gathered}
$$

and

$$
D_{i}=\frac{\partial A_{i}}{\partial \eta}=\frac{\partial}{\partial \eta}\left(\frac{d_{i}^{\eta}}{d_{i}^{\eta}+\kappa^{\eta}}\right)=\frac{d_{i}^{\eta} \kappa^{\eta}\left[\ln \left(d_{i}\right)-\ln (\kappa)\right]}{\left(d_{i}^{\eta}+\kappa^{\eta}\right)^{2}}
$$

Note:

For $D_{i}$

$$
\begin{aligned}
\frac{\partial}{\partial x}\left(a^{x}\right) & =a^{x} \ln (a) \Rightarrow \\
\frac{\partial}{\partial x}\left(\frac{a^{x}}{a^{x}+b^{x}}\right) & =\frac{a^{x} b^{x}[\ln (a)-\ln (b)]}{\left[a^{x}+b^{x}\right]^{2}}
\end{aligned}
$$

taking the partial derivatives yields 


$$
\begin{aligned}
\frac{\partial l(\boldsymbol{\theta})}{\partial \alpha} & =\frac{\partial}{\partial \alpha} \sum_{i=1}^{t}\left\{x_{i} \ln \left[\alpha+(1-\alpha) B_{i}\right]+\left(n_{i}-x_{i}\right) \ln \left[(1-\alpha)\left(1-B_{i}\right)\right]\right\} \\
& =\sum_{i=1}^{t}\left\{x_{i} \frac{1-B_{i}}{\alpha+(1-\alpha) B_{i}}+\left(n_{i}-x_{i}\right) \frac{(-1)\left(1-B_{i}\right)}{(1-\alpha)\left(1-B_{i}\right)}\right\} \\
& =\sum_{i=1}^{t}\left\{x_{i} \frac{1-B_{i}}{\alpha+(1-\alpha) B_{i}}-\left(n_{i}-x_{i}\right) \frac{\left(1-B_{i}\right)}{(1-\alpha)\left(1-B_{i}\right)}\right\} \\
& =\sum_{i=1}^{t}\left\{\frac{x_{i}\left(1-B_{i}\right)}{\alpha+(1-\alpha) B_{i}}-\frac{\left(n_{i}-x_{i}\right)}{(1-\alpha)}\right\} \\
\frac{\partial l(\boldsymbol{\theta})}{\partial \kappa} & =\frac{\partial}{\partial \kappa} \sum_{i=1}^{t}\left\{x_{i} \ln \left[\alpha+(1-\alpha) B_{i}\right]+\left(n_{i}-x_{i}\right) \ln \left[(1-\alpha)\left(1-B_{i}\right)\right]\right\} \\
& =\sum_{i=1}^{t}\left\{\frac{x_{i}(1-\alpha) C_{i}}{\alpha+(1-\alpha) B_{i}}+\frac{\left(n_{i}-x_{i}\right)(\alpha-1) C_{i}}{(1-\alpha)\left(1-B_{i}\right)}\right\} \\
& =\sum_{i=1}^{t}\left\{\frac{x_{i}(1-\alpha) C_{i}}{\alpha+(1-\alpha) B_{i}}-\frac{\left(n_{i}-x_{i}\right) C_{i}}{\left(1-B_{i}\right)}\right\}
\end{aligned}
$$

and

$$
\begin{aligned}
\frac{\partial l(\boldsymbol{\theta})}{\partial \eta} & =\frac{\partial}{\partial \eta} \sum_{i=1}^{t}\left\{x_{i} \ln \left[\alpha+(1-\alpha) B_{i}\right]+\left(n_{i}-x_{i}\right) \ln \left[(1-\alpha)\left(1-B_{i}\right)\right]\right\} \\
& =\sum_{i=1}^{t}\left\{\frac{x_{i}(1-\alpha) D_{i}}{\alpha+(1-\alpha) B_{i}}+\frac{\left(n_{i}-x_{i}\right)(\alpha-1) D_{i}}{(1-\alpha)\left(1-B_{i}\right)}\right\} \\
& =\sum_{i=1}^{t}\left\{\frac{x_{i}(1-\alpha) D_{i}}{\alpha+(1-\alpha) B_{i}}-\frac{\left(n_{i}-x_{i}\right) D_{i}}{\left(1-B_{i}\right)}\right\}
\end{aligned}
$$

Then, since $\hat{\kappa}$ and $\hat{\eta}$ cannot be solved analytically, we use the NewtonRaphson method to solve for $\boldsymbol{\theta}=(\alpha, \kappa, \eta)^{T}$

Recall that the Newton-Raphson method is given by

$$
\boldsymbol{\theta}^{(m+1)}=\boldsymbol{\theta}^{(m)}+\left.\left(-\frac{\partial^{2} l(\boldsymbol{\theta})}{\partial \boldsymbol{\theta} \partial \boldsymbol{\theta}^{T}}\right)^{-1} \frac{\partial l(\boldsymbol{\theta})}{\partial \boldsymbol{\theta}}\right|_{\boldsymbol{\theta}=\boldsymbol{\theta}^{(m)}}
$$


First, we compute the Hessian matrix. Since

$$
\begin{aligned}
& \frac{\partial l(\boldsymbol{\theta})}{\partial \alpha}=\sum_{i=1}^{t}\left\{\frac{x_{i}\left(1-B_{i}\right)}{\alpha+(1-\alpha) B_{i}}-\frac{\left(n_{i}-x_{i}\right)}{(1-\alpha)}\right\} \Rightarrow \\
& \frac{\partial^{2} l(\boldsymbol{\theta})}{\partial \alpha^{2}}=\frac{\partial}{\partial \alpha}\left(\frac{\partial l(\boldsymbol{\theta})}{\partial \alpha}\right)=\frac{\partial}{\partial \alpha}\left(\sum_{i=1}^{t}\left\{\frac{x_{i}\left(1-B_{i}\right)}{\alpha+(1-\alpha) B_{i}}-\frac{\left(n_{i}-x_{i}\right)}{(1-\alpha)}\right\}\right) \\
& =-\sum_{i=1}^{t}\left\{\frac{x_{i}\left(1-B_{i}\right)^{2}}{\left[\alpha+(1-\alpha) B_{i}\right]^{2}}+\frac{\left(n_{i}-x_{i}\right)}{(1-\alpha)^{2}}\right\} \\
& \frac{\partial^{2} l(\boldsymbol{\theta})}{\partial \kappa \partial \alpha}=\frac{\partial}{\partial \kappa}\left(\frac{\partial l(\boldsymbol{\theta})}{\partial \alpha}\right)=\frac{\partial}{\partial \kappa}\left(\sum_{i=1}^{t}\left\{\frac{x_{i}\left(1-B_{i}\right)}{\alpha+(1-\alpha) B_{i}}-\frac{\left(n_{i}-x_{i}\right)}{(1-\alpha)}\right\}\right) \\
& =\frac{\partial}{\partial \kappa}\left(\sum_{i=1}^{t}\left\{\frac{x_{i}\left(1-B_{i}\right)}{\alpha+(1-\alpha) B_{i}}\right\}\right) \\
& =\sum_{i=1}^{t}\left\{\frac{-x_{i} C_{i}}{\alpha+(1-\alpha) B_{i}}+\frac{x_{i}\left(1-B_{i}\right)(-1)\left(-\alpha C_{i}\right)}{\left[\alpha+(1-\alpha) B_{i}\right]^{2}}\right\} \\
& =-\sum_{i=1}^{t}\left\{\frac{x_{i} B_{i} C_{i}}{\left[\alpha+(1-\alpha) B_{i}\right]^{2}}\right\} \\
& \frac{\partial^{2} l(\boldsymbol{\theta})}{\partial \eta \partial \alpha}=\frac{\partial}{\partial \eta}\left(\frac{\partial l}{\partial \alpha}\right)=\frac{\partial}{\partial \eta}\left(\sum_{i=1}^{t}\left\{\frac{x_{i}\left(1-B_{i}\right)}{\alpha+(1-\alpha) B_{i}}-\frac{\left(n_{i}-x_{i}\right)}{(1-\alpha)}\right\}\right) \\
& =\frac{\partial}{\partial \eta}\left(\sum_{i=1}^{t}\left\{\frac{x_{i}\left(1-B_{i}\right)}{\alpha+(1-\alpha) B_{i}}\right\}\right) \\
& =-\sum_{i=1}^{t}\left\{\frac{x_{i} B_{i} D_{i}}{\left[\alpha+(1-\alpha) B_{i}\right]^{2}}\right\}
\end{aligned}
$$

since the only difference between $\frac{\partial^{2} l(\boldsymbol{\theta})}{\partial \kappa \partial \alpha}$ and $\frac{\partial^{2} l(\boldsymbol{\theta})}{\partial \eta \partial \alpha}$ is the partial derivatives with respect to $B_{i}$ 
Now, note that

$$
\begin{gathered}
E_{i}=\frac{\partial C_{i}}{\partial \kappa}=\frac{\partial}{\partial \kappa}\left(\frac{-\eta d_{i}^{\eta} \kappa^{\eta-1}}{\left(d_{i}^{\eta}+\kappa^{\eta}\right)^{2}}\right) \\
=\left(\frac{-\eta(\eta-1) d_{i}^{\eta} \kappa^{\eta-1}}{\left(d_{i}^{\eta}+\kappa^{\eta}\right)^{2}}+\frac{2 \eta^{2} d_{i}^{\eta} \kappa^{2(\eta-1)}}{\left(d_{i}^{\eta}+\kappa^{\eta}\right)^{3}}\right) \\
=\left(\frac{2 \eta^{2} d_{i}^{\eta} \kappa^{2(\eta-1)}}{\left(d_{i}^{\eta}+\kappa^{\eta}\right)^{3}}-\frac{\eta(\eta-1) d_{i}^{\eta} \kappa^{\eta-1}}{\left(d_{i}^{\eta}+\kappa^{\eta}\right)^{2}}\right) \\
\frac{\partial^{2} l(\boldsymbol{\theta})}{\partial \kappa^{2}}=\frac{\partial}{\partial \kappa}\left(\frac{\partial l(\boldsymbol{\theta})}{\partial \kappa}\right)=\frac{\partial}{\partial \kappa}\left(\sum_{i=1}^{t}\left\{\frac{x_{i}(1-\alpha) C_{i}}{\alpha+(1-\alpha) B_{i}}-\frac{\left(n_{i}-x_{i}\right) C_{i}}{\left(1-B_{i}\right)}\right\}\right) \\
=\sum_{i=1}^{t}\left\{\frac{(1-\alpha) x_{i} E_{i}}{\alpha+(1-\alpha) B_{i}}-\frac{\alpha(1-\alpha) x_{i} C_{i}^{2}}{\left[\alpha+(1-\alpha) B_{i}\right]^{2}}-\frac{\left(n_{i}-x_{i}\right) E_{i}}{\left(1-B_{i}\right)}-\frac{\left(n_{i}-x_{i}\right) C_{i}^{2}}{\left(1-B_{i}\right)^{2}}\right\}
\end{gathered}
$$

Similarly, define

$$
\begin{aligned}
F_{i} & =\frac{\partial C_{i}}{\partial \eta}=\frac{\partial}{\partial \eta}\left(\frac{-\eta d_{i}^{\eta} \kappa^{\eta-1}}{\left(d_{i}^{\eta}+\kappa^{\eta}\right)^{2}}\right) \\
& =\frac{d_{i}^{\eta} \kappa^{\eta-1}\left\{\eta\left(d_{i}^{\eta}-\kappa^{\eta}\right)\left[\ln \left(d_{i}\right)-\ln (\kappa)\right]-d_{i}^{\eta}-\kappa^{\eta}\right\}}{\left(d_{i}^{\eta}+\kappa^{\eta}\right)^{3}}
\end{aligned}
$$

then,

$$
\begin{aligned}
\frac{\partial^{2} l(\boldsymbol{\theta})}{\partial \eta \partial \kappa} & =\frac{\partial}{\partial \eta}\left(\frac{\partial l}{\partial \kappa}\right)=\frac{\partial}{\partial \eta}\left(\sum_{i=1}^{t}\left\{\frac{x_{i}(1-\alpha) C_{i}}{\alpha+(1-\alpha) B_{i}}-\frac{\left(n_{i}-x_{i}\right) C_{i}}{\left(1-B_{i}\right)}\right\}\right) \\
& =\sum_{i=1}^{t}\left\{\frac{(1-\alpha) x_{i} F_{i}}{\alpha+(1-\alpha) B_{i}}-\frac{\alpha(1-\alpha) x_{i} C_{i} D_{i}}{\left[\alpha+(1-\alpha) B_{i}\right]^{2}}-\frac{\left(n_{i}-x_{i}\right) F_{i}}{\left(1-B_{i}\right)}-\frac{\left(n_{i}-x_{i}\right) C_{i} D_{i}}{\left(1-B_{i}\right)^{2}}\right\}
\end{aligned}
$$

(since $\frac{\partial^{2} l(\boldsymbol{\theta})}{\partial \eta \partial \kappa}$ and $\frac{\partial^{2} l(\boldsymbol{\theta})}{\partial \kappa^{2}}$ only differ in terms of the partial derivatives of $B_{i}$ and $\left.C_{i}\right)$

Finally, letting

$$
\begin{aligned}
G_{i} & =\frac{\partial D_{i}}{\partial \eta}=\frac{\partial}{\partial \eta}\left(\frac{d_{i}^{\eta} \kappa^{\eta}\left[\ln \left(d_{i}\right)-\ln (\kappa)\right]}{\left(d_{i}^{\eta}+\kappa^{\eta}\right)^{2}}\right) \\
& =\frac{d_{i}^{\eta} \kappa^{\eta}\left(d_{i}^{\eta}-\kappa^{\eta}\right)\left[\ln \left(d_{i}\right)-\ln (\kappa)\right]^{2}}{\left(d_{i}^{\eta}+\kappa^{\eta}\right)^{3}}
\end{aligned}
$$


we get

$$
\begin{aligned}
\frac{\partial^{2} l(\boldsymbol{\theta})}{\partial \eta^{2}} & =\frac{\partial}{\partial \eta}\left(\frac{\partial l(\boldsymbol{\theta})}{\partial \eta}\right)=\frac{\partial}{\partial \eta}\left(\sum_{i=1}^{t}\left\{\frac{x_{i}(1-\alpha) D_{i}}{\alpha+(1-\alpha) B_{i}}-\frac{\left(n_{i}-x_{i}\right) D_{i}}{\left(1-B_{i}\right)}\right\}\right) \\
& =\sum_{i=1}^{t}\left\{\frac{(1-\alpha) x_{i} G_{i}}{\alpha+(1-\alpha) B_{i}}-\frac{\alpha(1-\alpha) x_{i} D_{i}^{2}}{\left[\alpha+(1-\alpha) B_{i}\right]^{2}}-\frac{\left(n_{i}-x_{i}\right) G_{i}}{\left(1-B_{i}\right)}-\frac{\left(n_{i}-x_{i}\right) D_{i}^{2}}{\left(1-B_{i}\right)^{2}}\right\}
\end{aligned}
$$




\section{A.2 Number of dose levels required for optimal designs for BMR and SNCR}

In his paper in 1953, Chernoff gave the following theorem.

Theorem 1 Chernoff's theorem

Suppose that we have $u$ estimators of our interest, which may depend on $\boldsymbol{\theta}=\left(\theta_{1}, \ldots, \theta_{k}\right)^{T}$.

If $R$ is closed and bounded, there is an element $\tilde{X}$ of $R$ which minimizes $v_{u}(X)=x^{11}+\ldots+x^{u u}$ and which is a convex linear combination of $t \leq$ $k+\ldots+(k-u+1)$ elements $\tilde{X}_{1}, \ldots, \tilde{X}_{t}$ of $R_{1}$. Furthermore, $\tilde{X}_{1}, \ldots, \tilde{X}_{t}$ may be chosen so that $v_{u}(X)$ is a continuous function at $X=\tilde{X}$ with respect to the topology of the convex set generated by $\tilde{X}_{1}, \ldots, \tilde{X}_{t}$.

where:

- $\underset{k(k+1) / 2}{R_{1}}$ is a set of vectors corresponding to the information matrix $\boldsymbol{X}\left(\boldsymbol{\theta}^{(0)}\right)$, and

- $R$ is a convex hull of $R_{1}$, which represents the set of information matrices of the class of mixed experiments.

Here, we are interested in estimating the excess risk $\pi_{0}$, which depends on the Hill model parameters $\boldsymbol{\theta}$.

The Chernoff's theorem states that if the information matrix $\boldsymbol{X}_{(\hat{\boldsymbol{\theta}})}$ is closed and bounded at any $d \in[0, D]$ under any given set of $\boldsymbol{\theta} \in \boldsymbol{\Theta}$, then there 
exists a sef of dose levels $\boldsymbol{d}$, which minimizes the variance of the estimator $\hat{\pi}_{0}$. Furthermore, the theorem tells us that such an optimal design would require three dose levels.

Proof:

At a dose level $d^{\prime} \in[0, D]$, the information matrix is given by:

$$
\frac{n P_{r}\left(d^{\prime}\right) P_{s}\left(d^{\prime}\right)}{P\left(d^{\prime}\right)\left[1-P\left(d^{\prime}\right)\right]} \propto \frac{P_{r}\left(d^{\prime}\right) P_{s}\left(d^{\prime}\right)}{P\left(d^{\prime}\right)\left[1-P\left(d^{\prime}\right)\right]}
$$

where $P_{r}\left(d^{\prime}\right)$ and $P_{s}\left(d^{\prime}\right)$ represent first partial derivatives of $P\left(d^{\prime}\right)$ with respect to $\theta_{i}$.

First, note that the denominator $P(d)[1-P(d)]$ at the boundary of $d$ (i.e., at 0 and $D)$ are: $\alpha(1-\alpha)$ and $(1-\alpha)\left[\alpha+(1-\alpha) \frac{D^{\eta}}{D^{\eta}+\kappa^{\eta}}\right]\left(\frac{\kappa^{\eta}}{D^{\eta}+\kappa^{\eta}}\right)$, respectively.

Taking a partial derivative with respect to $d$ is given by:

$$
\frac{\partial P(d)[1-P(d)]}{\partial d}=\frac{(\alpha-1) \eta \kappa^{\eta} d^{\eta-1}\left[(2 \alpha-1) \kappa^{\eta}+d^{\eta}\right]}{\left(d^{\eta}+\kappa^{\eta}\right)^{3}}
$$

which is continuous for any $d \in[0, D]$. In other words, $P(d)[1-P(d)]$ is closed and bounded.

Second, note that the partial derivative with respect to $\alpha$ is given by:

$$
P_{\alpha}(d)=\frac{\kappa^{\eta}}{d^{\eta}+\kappa^{\eta}}
$$

which is a monotonic function in $d$, bounded by $\frac{\kappa^{\eta}}{D^{\eta}+\kappa^{\eta}}$ and 1 . Hence $P_{\alpha}(d)$ is closed and bounded.

Third, the partial derivative with respect to $\kappa$ is given by:

$$
P_{\kappa}(d)=-\frac{(1-\alpha) \eta d^{\eta} \kappa^{\eta-1}}{\left(d^{\eta}+\kappa^{\eta}\right)^{2}}
$$


At $d=0$ and $D, P_{\kappa}(d)$ is 0 and $-\frac{(1-\alpha) \eta D^{\eta} \kappa^{\eta-1}}{\left(D^{\eta}+\kappa^{\eta}\right)^{2}}$.

Furthermore, taking the partial derivative with respect to $d$, we get:

$$
\frac{\partial P_{\kappa}(d)}{\partial d}=-\frac{(1-\alpha) \eta^{2} \kappa^{\eta-1}}{\left(\kappa^{\eta}+d^{\eta}\right)^{3}}\left[d^{\eta-1} \kappa^{\eta}-d^{2 \eta-1}\right]
$$

Solving (20) gives that the extremum is obtained at $d=\kappa$, hence $P_{\kappa}(d)$ is closed and bounded by $-\frac{(1-\alpha) \eta}{4 \kappa}$ and 0 .

Fourth, the partial derivative with respect to $\eta$ is given by:

$$
P_{\eta}(d)=\frac{(1-\alpha) d^{\eta} \kappa^{\eta}[\ln (d)-\ln (\kappa)]}{\left(d^{\eta}+\kappa^{\eta}\right)^{2}}
$$

At $d=0$ and $D, P_{\eta}(d)$ is 0 and $-\frac{(1-\alpha) D^{\eta} \kappa^{\eta}[\ln (D)-\ln (\kappa)]}{\left(D^{\eta}+\kappa^{\eta}\right)^{2}}$

taking the partial derivative with respect to $d$ gives $\frac{\partial P_{\eta}(d)}{\partial d}=\frac{(1-\alpha) \kappa^{\eta d^{\eta-1}}}{\left(d^{\eta}+\kappa^{\eta}\right)^{3}}\left\{(\eta[\ln (d)-\ln (\kappa)]+1)\left(\kappa^{\eta}+d^{\eta}\right)-2 \eta d^{\eta}[\ln (d)-\ln (\kappa)]\right\}$

where extrema cannot be solved explicitly. However, since $\frac{\partial P_{\eta}(d)}{\partial d}$ is continuous for any $d \in[0, D], P_{\eta}(d)$ is closed and bounded.

Since $P(d)[1-P(d)], P_{\alpha}(d), P_{\kappa}(d)$, and $P_{\eta}(d)$ are all closed and bounded, their function $\boldsymbol{X}_{(\hat{\boldsymbol{\theta}})}$ at any $d \in[0, D]$ is closed and bounded as well. 


\section{A.3 The Identifiability and the Regularity Conditions}

\section{A.3.1 Identifiability}

The following section provides the proofs for deriving the approximate asymptotic variance of the estimated excess risk $\hat{\pi}_{0}$. Note that definitions, theorems, lemmas, and corollaries are quoted from Krewski and Ryzin (1981)

\section{Definition:}

A dose response model $P(d)=P(d \mid \boldsymbol{\theta})$ is said to be identifiable if $P(d \mid \boldsymbol{\theta})=$ $P\left(d \mid \boldsymbol{\theta}^{\prime}\right) \forall d \geq 0$ implies that $\theta=\theta^{\prime}$. If the model is not identifiable, then the maximum likelihood estimator of $\theta$ discussed below may not be unique.

For the Hill model with the completely independent background, we can prove its identifiability as follows

Proof:

Let

$$
P(d \mid \boldsymbol{\theta})=\alpha+(1-\alpha)\left(\frac{d^{\eta}}{\kappa^{\eta}+d^{\eta}}\right)=\alpha^{\prime}+\left(1-\alpha^{\prime}\right)\left(\frac{d^{\eta^{\prime}}}{\kappa^{\prime \eta^{\prime}}+d^{\eta^{\prime}}}\right)=P\left(d \mid \boldsymbol{\theta}^{\prime}\right)
$$

Then it is clear that we must have $\alpha=\alpha^{\prime}$, since at $d=0$,

$$
P(0 \mid \boldsymbol{\theta})=\alpha=\alpha^{\prime}=P\left(0 \mid \boldsymbol{\theta}^{\prime}\right)
$$

Now, note that

$$
\begin{aligned}
\left(\frac{d^{\eta}}{\kappa^{\eta}+d^{\eta}}\right) & =\left(\frac{d^{\eta^{\prime}}}{\kappa^{\prime \eta^{\prime}}+d^{\eta^{\prime}}}\right) \Rightarrow \\
\frac{\kappa^{\eta}}{d^{\eta}} & =\frac{\kappa^{\prime \eta^{\prime}}}{d^{\eta^{\prime}}} \Rightarrow \\
\kappa^{\eta} & =d^{\left(\eta-\eta^{\prime}\right)} \kappa^{\prime \eta^{\prime}}
\end{aligned}
$$

which implies that $\eta=\eta^{\prime}$. This, in turn, proves that $\kappa=\kappa^{\prime}$. Hence the 
model is identifiable.

\section{A.3.2 Regularity conditions}

The following regularity conditions are required to establish the desired asymptotic properties of the maximum likelihood estimator $\hat{\boldsymbol{\theta}}$

- $\mathrm{C} 1$ :

$$
\lim _{n \rightarrow \infty} \frac{n_{i}}{n}=c_{i}\left(0 \leq c_{0}<1 ; 0<c_{i}<1 \text { for } i=1, \ldots, t\right)
$$

where $c_{0}$ represents the proportion of allocation for the control group.

- $\mathrm{C} 2: P(d \mid \boldsymbol{\theta})$ is identifiable

- C3: The information matrix $\Sigma^{-1}=\frac{\boldsymbol{X}}{n}$ defined by

$$
\Sigma^{-1}=\sum_{i=1}^{t} \frac{c_{i} P_{r}\left(d_{i}\right) P_{s}\left(d_{i}\right)}{P\left(d_{i}\right)\left[1-P^{*}\left(d_{i}\right)\right]} \text { for } r, s=1, \ldots, k
$$

is positive definite.

- C4: For all $d \geq 0$, the second partial derivatives $P_{r, s}\left(d_{i}\right)$ for $r, s=$ $1, \ldots, k$ are continuous for all $\boldsymbol{\theta}=\boldsymbol{\Theta}$.

- C5: $P(d \mid \boldsymbol{\theta})$ is continuously differentiable in $d$ and $\theta$ for all $d>0$ and $\boldsymbol{\theta}=\Theta$.

\section{Verification:}

First, $\mathrm{C} 1$ is satisfied as we set $n_{i} \approx c_{i} n$ where $c_{i}$ 's are determined apriori to the experiment, and $\mathrm{C} 2$ has been verified in section A.3.1.

Second, C4 is also satisfied as 


$$
\begin{aligned}
& \frac{\partial^{2} P(d)}{\partial \alpha^{2}}=0 ; \frac{\partial^{2} P(d)}{\partial \alpha \partial \kappa}=-C ; \frac{\partial^{2} P(d)}{\partial \alpha \partial \eta}=-D \\
& \frac{\partial^{2} P(d)}{\partial \kappa^{2}}=(1-\alpha) E ; \frac{\partial^{2} P(d)}{\partial \kappa \partial \eta}=(1-\alpha) F ; \frac{\partial^{2} P(d)}{\partial \eta^{2}}=(1-\alpha) G
\end{aligned}
$$

where $C$ to $G$ are given in (15) (16) (17) (18) (19), which are clearly continuous for all $\boldsymbol{\theta}=\boldsymbol{\Theta}$.

Finally, C5 holds as

$$
\begin{aligned}
& \frac{\partial P(d)}{\partial d}=\frac{\eta \kappa^{\eta} d^{\eta-1}}{(\kappa+d)^{2}} \\
& \frac{\partial P(d)}{\partial \alpha}=\frac{\kappa^{\eta}}{\kappa^{\eta}+d^{\eta}}=1-B \\
& \frac{\partial P(d)}{\partial \kappa}=-(1-\alpha) \frac{\eta d_{i}^{\eta} \kappa^{\eta-1}}{\left(\kappa^{\eta}+d_{i}^{\eta}\right)^{2}}=(1-\alpha) C \\
& \frac{\partial P(d)}{\partial \eta}=(1-\alpha) \frac{d_{i}^{\eta} \kappa^{\eta}\left[\ln \left(d_{i}\right)-\ln (\kappa)\right]}{\left(\kappa^{\eta}+d_{i}^{\eta}\right)^{2}}=(1-\alpha) D
\end{aligned}
$$

are continuous for all $d>0$ and $\boldsymbol{\theta}=\boldsymbol{\Theta}$.

The verification of C3 is more complicated; however, Krewski and Ryzin (1981) provide the following lemmas using the Chebyshev set.

Definition:

A set of functions $\left\{\phi_{r}(d) ; r=1, \ldots, k\right\}$ is said to form a Chebyshev set on $[0, D]$ if $\phi(d ; \boldsymbol{a})=\sum a_{r} \phi_{r}(d)$ has at most $k-1$ zeros in $[0, D]$ for all $a=\left(a_{1}, \ldots, a_{k}\right)^{T}$.

\section{Lemma2:}

The set of functions $\left\{\phi_{r}(d) ; r=1, \ldots, k\right\}$ forms a Chebyshev set on $[0, D]$ if and only if the matrix $\left\langle\left(\phi_{r}\left(d_{i}\right)\right)\right\rangle(r, i=1, \ldots, k)$ is of full rank for every set 
of $k$ distinct points $\left\{d_{i}\right\}$ in $[0, D]$.

Lemma3:

The informatix $\Sigma^{-1}$ in C3 is positive definite provided that $\left\{\frac{\partial P(d)}{\partial \theta_{r}} ; r=1, \ldots, k\right\}$ forms a Chebyshev set on $\left[0, d_{I+1}\right]$ where $I+1>k$.

In other words, the information matrix $\boldsymbol{\Sigma}^{-1}$ is positive definite if we can prove that the matrix $\left\langle\left(\frac{\partial P\left(d_{i}\right)}{\partial \theta_{r}}\right)\right\rangle(r, i=1,2,3)$ is of full rank for every set of $k$ distinct points $\left\{d_{i}\right\}$ in $\left[0, d_{t}\right]$.

The proof of the above statement is given as follows:

Proof:

We have

$$
\left\langle\left(\frac{\partial P\left(d_{i}\right)}{\partial \theta_{r}}\right)\right\rangle=\left[\begin{array}{lll}
\frac{\partial P\left(d_{1}\right)}{\partial \alpha} & \frac{\partial P\left(d_{1}\right)}{\partial \kappa} & \frac{\partial P\left(d_{1}\right)}{\partial \eta} \\
\frac{\partial P\left(d_{2}\right)}{\partial \alpha} & \frac{\partial P\left(d_{2}\right)}{\partial \kappa} & \frac{\partial P\left(d_{2}\right)}{\partial \eta} \\
\frac{\partial P\left(d_{3}\right)}{\partial \alpha} & \frac{\partial P\left(d_{3}\right)}{\partial \kappa} & \frac{\partial P\left(d_{3}\right)}{\partial \eta}
\end{array}\right] ;\left\{d_{i}\right\} \in\left[0, d_{t}\right]
$$

In order to show the above matrix is of full rank, we are required to prove that $c_{1} \frac{\partial P\left(d_{1}\right)}{\partial \boldsymbol{\theta}}+c_{2} \frac{\partial P\left(d_{2}\right)}{\partial \boldsymbol{\theta}}+c_{3} \frac{\partial P\left(d_{3}\right)}{\partial \boldsymbol{\theta}}=\mathbf{0}$ implies that $c_{1}=c_{2}=c_{3}=0$.

Suppose now that we have $c_{1} \frac{\partial P\left(d_{1}\right)}{\partial \alpha}+c_{2} \frac{\partial P\left(d_{2}\right)}{\partial \alpha}=c_{3} \frac{\partial P\left(d_{3}\right)}{\partial \alpha}$ for a given set of $\boldsymbol{\theta} \in \boldsymbol{\Theta}$ and $d_{i} \in\left[0, d_{t}\right]$, where at least one of the $c_{i}$ are nonzero. That is, we have

$$
\begin{aligned}
c_{1} \frac{\kappa^{\eta}}{\kappa^{\eta}+d_{1}^{\eta}}+c_{2} \frac{\kappa^{\eta}}{\kappa^{\eta}+d^{\eta}} & =c_{3} \frac{\kappa^{\eta}}{\kappa^{\eta}+d^{\eta}} \Rightarrow \\
\frac{c_{1}}{\kappa^{\eta}+d_{1}^{\eta}}+\frac{c_{2}}{\kappa^{\eta}+d^{\eta}} & =\frac{c_{3}}{\kappa^{\eta}+d^{\eta}} \quad(*)
\end{aligned}
$$


Now, if the matrix $\left\langle\left(\frac{\partial P\left(d_{i}\right)}{\partial \theta_{r}}\right)\right\rangle$ is not of full rank, we must have, for the above $c_{1}, c_{2}$, and $c_{3}$,

$$
\begin{aligned}
c_{1} \frac{\partial P\left(d_{1}\right)}{\partial \kappa}+c_{2} \frac{\partial P\left(d_{2}\right)}{\partial \kappa} & =c_{3} \frac{\partial P\left(d_{3}\right)}{\partial \kappa} \Rightarrow \\
c_{1} \frac{d_{1}^{\eta}}{\left(\kappa^{\eta}+d_{1}^{\eta}\right)^{2}}+c_{2} \frac{d_{2}^{\eta}}{\left(\kappa^{\eta}+d_{2}^{\eta}\right)^{2}} & =c_{3} \frac{d_{3}^{\eta}}{\left(\kappa^{\eta}+d_{3}^{\eta}\right)^{2}} \Rightarrow \\
\frac{c_{1}}{\kappa^{\eta}+d_{1}^{\eta}}\left(\frac{d_{1}^{\eta}}{\kappa^{\eta}+d_{1}^{\eta}}\right)+\frac{c_{2}}{\kappa^{\eta}+d^{\eta}}\left(\frac{d_{2}^{\eta}}{\kappa^{\eta}+d_{2}^{\eta}}\right) & =\frac{c_{3}}{\kappa^{\eta}+d^{\eta}}\left(\frac{d_{3}^{\eta}}{\kappa^{\eta}+d_{3}^{\eta}}\right)
\end{aligned}
$$

Since $-(1-\alpha) \frac{\eta d_{i}^{\eta} \kappa^{\eta-1}}{\left(\kappa^{\eta}+d_{i}^{\eta}\right)^{2}}=\left[-(1-\alpha) \kappa^{\eta-1} \eta\right] \frac{d_{i}^{\eta}}{\left(\kappa^{\eta}+d_{i}^{\eta}\right)^{2}}$, the term in the square bracket is a constant. This implies that we must have $d_{1}=d_{2}=d_{3}$, which cannot be the case. Hence, $c_{1} \frac{\partial P\left(d_{1}\right)}{\partial \boldsymbol{\theta}}+c_{2} \frac{\partial P\left(d_{2}\right)}{\partial \boldsymbol{\theta}}+c_{3} \frac{\partial P\left(d_{3}\right)}{\partial \boldsymbol{\theta}}=\mathbf{0}$ implies that $c_{1}=c_{2}=c_{3}=0$ and the proof is complete.

\section{Theorem1:}

Under C1-C4, the maximum likelihood equations $\partial \Pi / \partial \theta=0$ have a unique root $\hat{\theta}$ with probability one, which is strongly consistent for $\theta$.

Since $P(d \mid \boldsymbol{\theta})$ is a continuous function of $\theta$ it follows from Theorem 1 that $\hat{\pi}(d)=\hat{P}(d)-\hat{P}(0)$ is a strongly consistent estimator of $\pi(d)$, where $\hat{P}(d)=\hat{P}(d \mid \boldsymbol{\theta})$. Similarly, $\hat{d}^{*}=\hat{\pi}^{-1}(\pi)$ is a strongly consistent estimator of $\hat{d}^{*}$ whenever C5 holds.

Theorem2:

Under $\mathrm{C} 1-\mathrm{C} 4, \sqrt{n}(\hat{\boldsymbol{\theta}}-\boldsymbol{\theta})$ is normally distributed with mean 0 and covariance matrix $\Sigma$.

Corollary: 
Under C1-C5, $\sqrt{n}(\hat{\pi}(d)-\pi(d))$ and $\sqrt{n}(\hat{d}-d)$ are asympototically normally distributed with zero means and

$$
\begin{aligned}
V[\hat{\pi}(d)] & =\boldsymbol{\Pi}^{T} \boldsymbol{X} \boldsymbol{\Pi} \\
V\left(\hat{d}^{*}\right) & =\left[\frac{\partial P\left(d^{*}\right)}{\partial d}\right]_{d=d^{*}}^{-2} V\left[\hat{\pi}\left(d^{*}\right)\right]
\end{aligned}
$$

where $\Pi^{T}=\left(\Pi_{1}, \ldots, \Pi_{k}\right) ; \Pi_{r}=P_{r}(d)-P_{r}(0)$. 


\section{A.4 Profile Likelihood based Confidence Interval for $\hat{P}(d)$}

Note that we have

$$
\begin{aligned}
\pi(d) & =(1-\alpha)\left(\frac{d^{\eta}}{d^{\eta}+\kappa^{\eta}}\right) \Leftrightarrow \\
\frac{\pi(d)}{1-\alpha} & =\frac{d^{\eta}}{d^{\eta}+\kappa^{\eta}} \Leftrightarrow \\
\frac{1-\alpha}{\pi(d)} & =1+\frac{\kappa^{\eta}}{d^{\eta}} \Leftrightarrow \\
\frac{1-\alpha}{\pi(d)}-1 & =\frac{\kappa^{\eta}}{d^{\eta}} \Leftrightarrow \\
\kappa & =d\left(\frac{1-\alpha}{\pi(d)}-1\right)^{1 / \eta}
\end{aligned}
$$

i.e., If we know $d, \pi(d), \alpha$, and $\eta$, we can determine $\kappa$.

Under some regularity conditions, we have the following condition

$$
2\left[l\left(\hat{\boldsymbol{\theta}}_{M L E}\right)-l\left(\hat{\boldsymbol{\theta}}_{0}\right)\right] \sim \chi_{m}^{2}
$$

Fixing $\left(\hat{\alpha}_{0}, \hat{\eta}_{0}\right)$ at $\left(\hat{\alpha}_{M L E}, \hat{\eta}_{M L E}\right)$ we have

$$
\hat{\boldsymbol{\theta}}_{0}=\left(\hat{\alpha}_{M L E}, \hat{\kappa}_{0}, \hat{\eta}_{M L E}\right) \text { with } m=1
$$

i.e.., we can test

$$
H_{0}: \boldsymbol{\theta}=\boldsymbol{\theta}_{0} \text { vs } H_{a}: \boldsymbol{\theta} \neq \boldsymbol{\theta}_{0}
$$

which basically is

$$
H_{0}: \kappa=\kappa_{0} \text { vs } H_{a}: \kappa \neq \kappa_{0}
$$

In other words, we accept $H_{0}$ if (at $\left.\alpha=0.05\right)$ 


$$
\begin{aligned}
2\left[l\left(\hat{\boldsymbol{\theta}}_{M L E}\right)-l\left(\hat{\boldsymbol{\theta}}_{0}\right)\right] & \leq \chi_{1}^{2}(0.95) \Leftrightarrow \\
l\left(\hat{\boldsymbol{\theta}}_{M L E}\right)-\frac{1}{2} \chi_{1}^{2}(0.95) & \leq l\left(\hat{\boldsymbol{\theta}}_{0}\right) \Leftrightarrow \\
l\left(\hat{\boldsymbol{\theta}}_{M L E}\right)-\frac{1}{2} \chi_{1}^{2}(0.95) & \leq l\left(\hat{\boldsymbol{\theta}}_{0}\right) \Leftrightarrow \\
l\left(\hat{\boldsymbol{\theta}}_{M L E}\right)-1.92 & \leq l\left(\hat{\boldsymbol{\theta}}_{0}\right)
\end{aligned}
$$

Then we can form a $90 \%$ C.I for $\hat{P}(d)$ based on profile likelihood method by choosing $\hat{\boldsymbol{\theta}}_{0}$ such that

$$
\begin{aligned}
l\left(\hat{\boldsymbol{\theta}}_{0}\right) & \geq l\left(\hat{\boldsymbol{\theta}}_{M L E}\right)-1.92 \text { and } \\
\hat{\boldsymbol{\theta}}_{0} & =\arg \max P(d \mid \hat{\boldsymbol{\theta}})
\end{aligned}
$$




\section{B Definitions}

Additional Risk: The additional probability of an effect at dose d compared to the probability of an effect in the absense of the dose. Mathematically,

$$
\text { Additional risk }=P(d)-P(0)
$$

Effective Dose (ED): A dose level that corresponds to a certain extra risk. For example, an effective dose of 50 denotes a dose level corresponding to 50 $\%$ extra risk.

Extra Risk: The probability of an effect at dose d given that no effect would have occurred in the absense of the dose (Crump, 1984) Mathematically,

$$
\text { Extra risk }=\frac{P(d)-P(0)}{1-P(0)}
$$

Excess Risk: See Additional Risk

Point of Departure (PoD): The dose that corresponds to the risk that is thought to be acceptable/tolerable from an experiment.

Reference Dose (RfD): The dose level to be used in the human exposure guidelines. It is often given by

$$
\mathrm{RfD}=\frac{P_{O D}}{U F}
$$

Uncertainty factor (UF): A safety factor to incorporate the inter- and intra-species variations from an observed experiment. It is used for derivation of the RfD.

Virtually Safe Dose: See Point of Departure 


\section{List of Acronyms}

BMD: Benchmark Dose

BMDL: Lower Confidence Limit on the Benchmark Dose

MDT: Maximum Dose Tested

MTD: Maximum Tolerated Dose

NOAEL: No Observed Adverse Effect Level

PoD: Point of Departure

RfD: Reference Dose

SNCD: Signal-to-Noise Crossover Dose

SNCR: Signal-to-Noise Crossover Risk

SNR: Signal-to-Noise Ratio

UF: Uncertainty Factor

VSD: Virtually Safe Dose 